\title{
A bijection for rooted maps on orientable surfaces
}

\author{
Guillaume Chapuy, LIX, École Polytechnique \\ and \\ Michel Marcus, LaBRI, Université Bordeaux 1 \\ and \\ Gilles Schaeffer, LIX, CNRS and École Polytechnique
}

\begin{abstract}
The enumeration of maps and the study of uniform random maps have been classical topics of combinatorics and statistical physics ever since the seminal work of Tutte in the sixties. Following the bijective approach initiated by Cori and Vauquelin in the eighties, we describe a bijection between rooted maps, or rooted bipartite quadrangulations, on a surface of genus $g$ and some simpler objects that generalize plane trees. Thanks to a rerooting argument, our bijection allows to compute the generating series of rooted maps on a surface of genus $g$ with respect to the number of edges, and to recover the asymptotic numbers of such maps.

Our construction allows to keep track in a bipartite quadrangulation of the distances of all vertices to a random basepoint. This is an analog for higher genus surfaces of the basic result on which were built the recent advances in the comprehension of the intrinsec geometry of large random planar maps, hopefully opening the way to the study of a model of continuum random surfaces of genus $g$.

Categories and Subject Descriptors: Combinatorics [G.2.1]: Enumeration; Graph theory

Additional Key Words and Phrases: Graphs on surfaces, trees, random discrete surfaces
\end{abstract}

\section{INTRODUCTION}

This article is concerned with the enumerative and probabilistic study of maps on orientable surfaces: rougly speaking a map of genus $g$ is a proper embedding of a graph in $\mathcal{S}_{g}$, the compact oriented surface of genus $g$ without boundary. Maps on the sphere $\mathcal{S}_{0}$, also known as planar maps, have been studied in enumerative combinatorics ever since the seminal papers of William T. Tutte in the sixties [Tut63], and our primary motivation is still to explore the unexpected enumerative properties of these objects: For instance, the number of rooted planar maps having $n$ edges is given by the simple formula

$$
\vec{q}_{0, n}=\frac{2 \cdot 3^{n}(2 n) !}{(n+2) ! n !}
$$

and the number of rooted maps of genus $g$ having $n$ edges satisfies

$$
\vec{q}_{g, n} \sim c_{g} \cdot n^{\frac{5}{2}(g-1)} \cdot 12^{n},
$$

when $n$ goes to infinity with $g$ fixed. The remarkable linear dependency in $g$ of the polynomial growth exponent of this asymptotic formula has been observed as a common pattern of many classes of maps in combinatorics [BC91; Gao93]. Formula (1) for the planar case was recovered also in the physics literature by

G.S. acknowledges financial support from the french ministery of research via grants ACI-MD Geocomp and ANR Sada. 

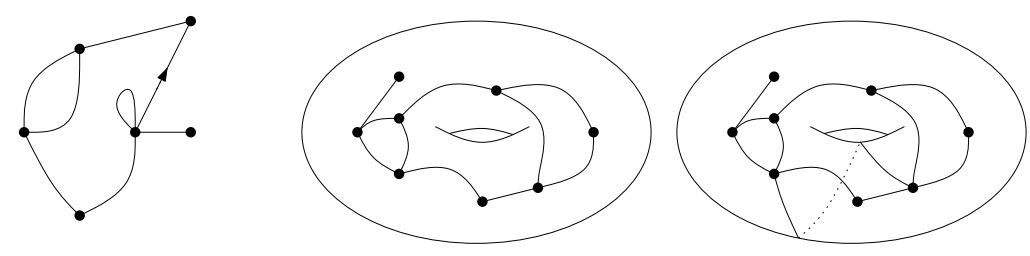

Fig. 1. A rooted planar map, a graph drawing on $\mathcal{S}_{1}$ and a map of genus 1.

mean of the so-called genus expansions of matrix integrals [BIPZ78], which also led in the 90's to independant derivations of the asymptotic formula (2) [DGZJ95, and reference therein]. Until now however it has remained an open problem to give a combinatorial explanation of the linear dependency in the genus [Ben91].

The aim of this paper is to describe a bijection (Theorem 1) which reformulates and extends to higher genus surfaces an earlier bijection of Cori and Vauquelin [CV81] between planar maps and well labelled trees. In the planar case, a rerooting argument then immediately leads to Formula 1 (Corollary 4). In higher genus, the combination of our bijection with a decomposition inspired from Wright's works on labeled graphs with fixed excess [Wri77; Wri78] leads to a simple combinatorial derivation of Formula (2) (Corollary 9), and to a new expression of the generating series for maps of genus $g$ with respect to the number of edges (Theorem 2). Classical results of singularity analysis allow then to recover easily the asymptotic formula (2), and provides a new expression to the constant $c_{g}$ in front of the formula (Corollary 9).

One motivation for the study of maps in statistical physics is the interpretation of random maps as discrete models of surfaces in the context of two-dimensional euclidean quantum gravity [ArDJ97]. From a probabilistic point of view, it is natural to wonder about the geometry of these random discrete surfaces. In the planar case our bijection was already used in [CS04] to show that the distance between two random vertices in a uniform random planar quadrangulation with $n$ faces is of order $n^{1 / 4}$, and more generally to study the process describing the number of vertices at distance $k$ of a vertex. This line of research has lead to remarkable progress in our understanding of the geometry of large random planar maps [BDFG04] and of their continuum limit [MM06; LG07; LGP07]. From this perspective we provide here the basic building block for the study of higher genus random discrete surfaces.

\section{MAPS, QUADRANGULATIONS AND $G$-TREES}

In this section we make precise our use of the graph terminology.

\subsection{Graph drawings and maps}

Let $\mathcal{S}_{g}$ denote the compact oriented surface of genus $g$ without boundary. A graph drawing on $\mathcal{S}_{g}$ is a proper embedding (without edge crossings) of a graph into $\mathcal{S}_{g}$. Multiple edges and loops are allowed. A graph drawing on $\mathcal{S}_{g}$ is a map of genus $g$ if moreover each face is homeomorphic to a disc, or equivalently, if the complement of the graph in $S_{g}$ is a union of disjoint simply connected components. In particular the graph underlying a map is necessarily connected. Maps of genus 0 are called 

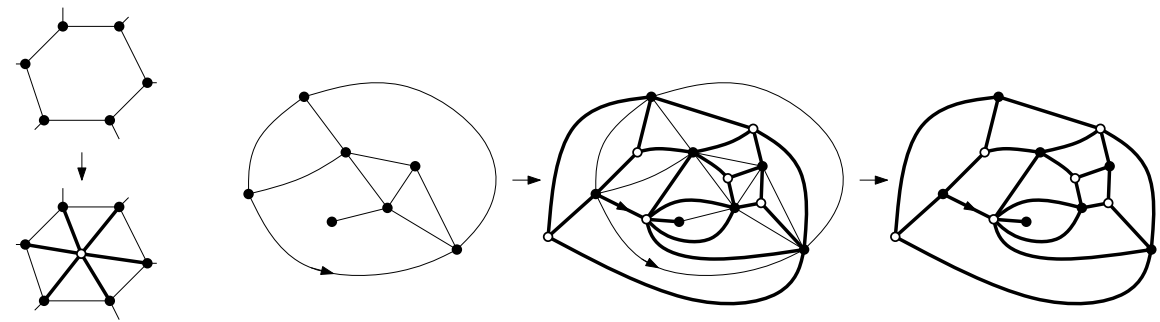

Fig. 2. The quadrangulation of a map: local construction and an exemple in the plane

planar maps. For enumerative purpose it is convenient to consider rooted maps, that is, pairs $\overrightarrow{\mathfrak{m}}=(\mathfrak{m}, e)$ where $\vec{e}$ is an arc of $\mathfrak{m}$ (an arc is an edge with an orientation). The arc $\vec{e}$ is called the root of $\overrightarrow{\mathfrak{m}}$, its origin is the root vertex of $\mathfrak{m}$ and the face on its right is the root face.

In the rest of this text, two maps are considered the same if there exists an orientation preserving homeomorphism of the embedding surface that sends one map onto the other (carrying roots if there are some). These (equivalence classes of) maps can also be defined in a purely combinatorial way: for instance a rooted map can be described by listing the local arrangement of arcs around each vertex using any numbering of these arcs. A modern account of the equivalence between topological and combinatorial descriptions of maps can be found in [MT01, Ch. 3].

To any rooted map $\overrightarrow{\mathfrak{m}}$ we associate an arbitrary fixed numbering of its edges (respectively arcs, vertices, corners, or faces) that starts with the root edge, which we call the canonical order of the edges (respectively arcs, vertices, corners, or faces) of $\overrightarrow{\mathfrak{m}}$. This numbering can for instance be given by the first visit in a depth first search turning counterclockwise around each vertex, or by any other fixed procedure: in what follows we will only need to invoke this canonical order to fix notations.

A fundamental result of the theory of surfaces is the Euler characteristic formula:

LEMMA 1. If a map of genus $g$ has $v$ vertices, $f$ faces and $n$ edges then

$$
v-n+f=2-2 g \text {. }
$$

The quantity $\chi=2-2 g$ is called the Euler characteristic of the map.

\subsection{Maps and bipartite quadrangulations}

The degree of a vertex is the number of edges incident to it, with the convention that a loop counts for two. The degree of a face is the number of edges incident to it, with the convention that an edge incident to the same face on both sides counts for two. A quadrangulation is a planar map having all faces of degree 4 . A map is bipartite if its vertices are colored in two colors in such a way that adjacent vertices have different colors (say black and white). By convention the color of the root vertex of a rooted bipartite map is always taken to be black. We recall two standard results of graph and map theory:

(1) All planar quadrangulations admit a bipartition.

(2) For all $n, v, f \geq 1$ and $g \geq 0$ with $v-n+f=2-2 g$, there is a bijection between maps of genus $g$ with $n$ edges, $v$ vertices and $f$ faces, and bipartite 

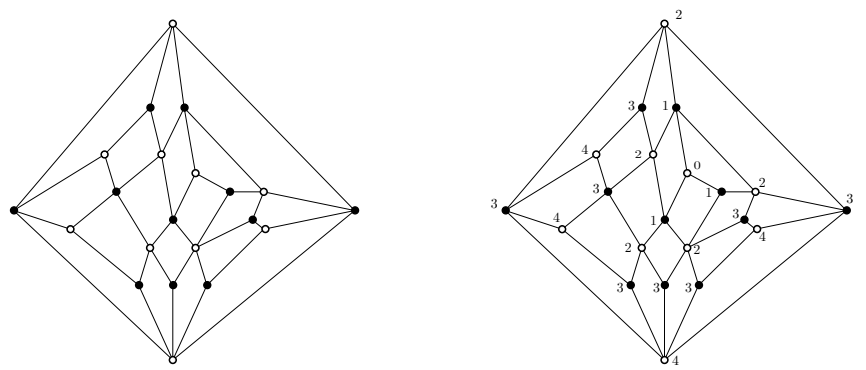

Fig. 3. A quadrangulation and its distance labeling

quadrangulations of genus $g$ with $n$ faces, $v$ black and $f$ white vertices, and $2 n$ edges. Idem between rooted maps and rooted bipartite quadrangulations.

The first result will not be used but is recalled to stress the fact that, a contrario, a similar statement does not hold for quadrangulations of genus $g$ : there exist non bipartite quadrangulations of genus $g$ for all $g \geq 1$.

The second result is based on a classical construction which we now briefly recall: Given a map $\mathfrak{m}$ with black vertices, triangulate each face from a new white vertex; the new bipartite edges form with black and white vertices a quadrangulation $\mathfrak{q}$, which is the bipartite quadrangulation associated to $\mathfrak{m}$. Conversely given a bipartite quadrangulation $\mathfrak{q}$ create inside each face $f$ a new edge connecting the two black vertices incident to $f$; these new edges form with the black vertices a map $\mathfrak{m}$ whose bipartite quadrangulation is $\mathfrak{q}$. To get a correspondence in the rooted case, a rerooting convention must be chosen: if $\mathfrak{m}$ has a root edge $e$, then let the root of $\mathfrak{q}$ be the arc of $\mathfrak{q}$ with origin the origin of $e$ and with the face containing $e$ on its right hand side. This correspondence is the reason why, although we concentrate in the rest of this text on bipartite quadrangulations, our results have implications for all maps.

\subsection{Quadrangulations, distance labelling and $g$-trees}

A pointed quadrangulation is a pair $\mathfrak{q}^{\bullet}=\left(\mathfrak{q}, v_{0}\right)$, where $v_{0}$ is a vertex of $\mathfrak{q}$, called the basepoint. The distance labelling of $\left(\mathfrak{q}, v_{0}\right)$ is a labelling of the vertices of $\mathfrak{q}$ by their distance to $v_{0}$ in the underlying graph: the label of a vertex $v$ is the minimum number of edges of a path returning from $v$ to $v_{0}$. Observe that in a pointed bipartite quadrangulation the vertices with the same color as the basepoint are exactly the vertices at even distance of it. In particular two adjacent vertices have labels that differ by one in the distance labelling, and the faces are of two types depending whether the cycle of incident labels is of the form $(i-1, i, i-1, i)$ or $(i-1, i, i+1)$ for some $i \geq 1$.

A plane tree is a planar map with only one face. In particular a planar tree contains no simple cycle of edges, and the definition of rooted planar trees agrees with the usual recursive combinatorial definition of ordered trees: a rooted plane tree can be uniquely decomposed into a root vertex and a (possibly empty) ordered sequence of rooted planar trees. By extension, we call $g$-tree a map of genus $g$ with one face. Observe however that for $g \geq 1$, any $g$-tree contains simple cycles of edges. 

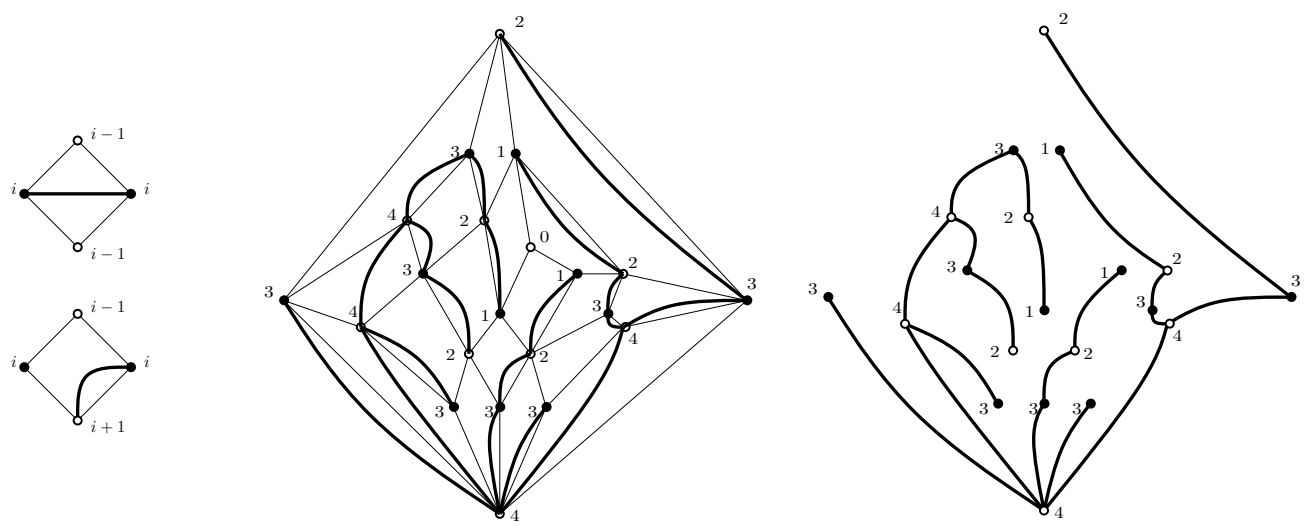

Fig. 4. The mapping $\phi$ : local rules and an example

\section{EMBEDDED $G$-TREES AND THE OPENING BIJECTION}

A map is called embedded if its vertices are given integer labels that differ at most by one along every edge, the root vertex (if the map is rooted) having label 1. A map is well labeled if moreover the support of labels is an interval of the form $(1, m)$. In other terms a well labeled map is an embedded map with minimum label 1.

\subsection{The opening of a pointed bipartite quadrangulation}

Given a pointed bipartite quadrangulation $\left(\mathfrak{q}, v_{0}\right)$ of genus $g$ having $n$ faces, we define a mapping $\phi$, the opening, which we will later claim produces a well labeled $g$-tree with $n$ edges (see Figure 4 ):

- Label vertices of $\mathfrak{q}$ according to their distance to $v_{0}$. Recall that the extremities of each edge have labels that differ by one, so that the labels of vertices on the border of a face form either a cycle $(i-1, i, i-1, i)$ or a cycle $(i-1, i, i+1, i)$.

-Create a graph drawing $\phi\left(\mathfrak{q}, v_{0}\right)$ on $\mathcal{S}_{g}$ with vertex set the vertices of $\mathfrak{q}$ except $v_{0}$ and with one edge $e_{f}$ for each face $f$ of $\mathfrak{q}$, which connects the two corners of $f$ that have a vertex label larger than their predecessor in clockwise direction around $f$ : in a face with border $(i-1, i, i-1, i)$ the resulting edge has label $(i, i)$, while in a face with border $(i-1, i, i+1, i)$ it has label $(i, i+1)$.

Obviously the labels of the vertices of $\phi\left(\mathfrak{q}, v_{0}\right)$ are positive and the variations along its edges belong to $\{+1,0,-1\}$, so that $\phi\left(\mathfrak{q}, v_{0}\right)$ is well labelled. More interesting is the following lemma, which proves that $\phi\left(\mathfrak{q}, v_{0}\right)$ is a well labelled $g$-tree.

LEMMA 2. The graph drawing $\phi\left(\mathfrak{q}, v_{0}\right)$ on $\mathcal{S}_{g}$ has a unique and simply connected face.

ProOF. We give here a quick proof which requires some familiarity with graph on surfaces, and postpone a more explicit proof to the appendix. The graph drawing $\mathfrak{q}^{\prime}=\mathfrak{q} \cup \phi\left(\mathfrak{q}, v_{0}\right)$ is obviously a map: each face of $\mathfrak{q}$ is divided in $\mathfrak{q}^{\prime}$ into 2 smaller simply connected faces by an edge of $\phi\left(\mathfrak{q}, v_{0}\right)$ (see Figure 4 ). Draw a fake vertex $v_{f}$ in each face $f$ of $\mathfrak{q}^{\prime}$, label it by the smallest label on the border of $f$, and connect these fake vertices by one fake edge for each edge of $\mathfrak{q}$ : an edge $e$ of $\mathfrak{q}$ with label $(i, i+1)$ that separates faces $f$ (on its right hand side) 

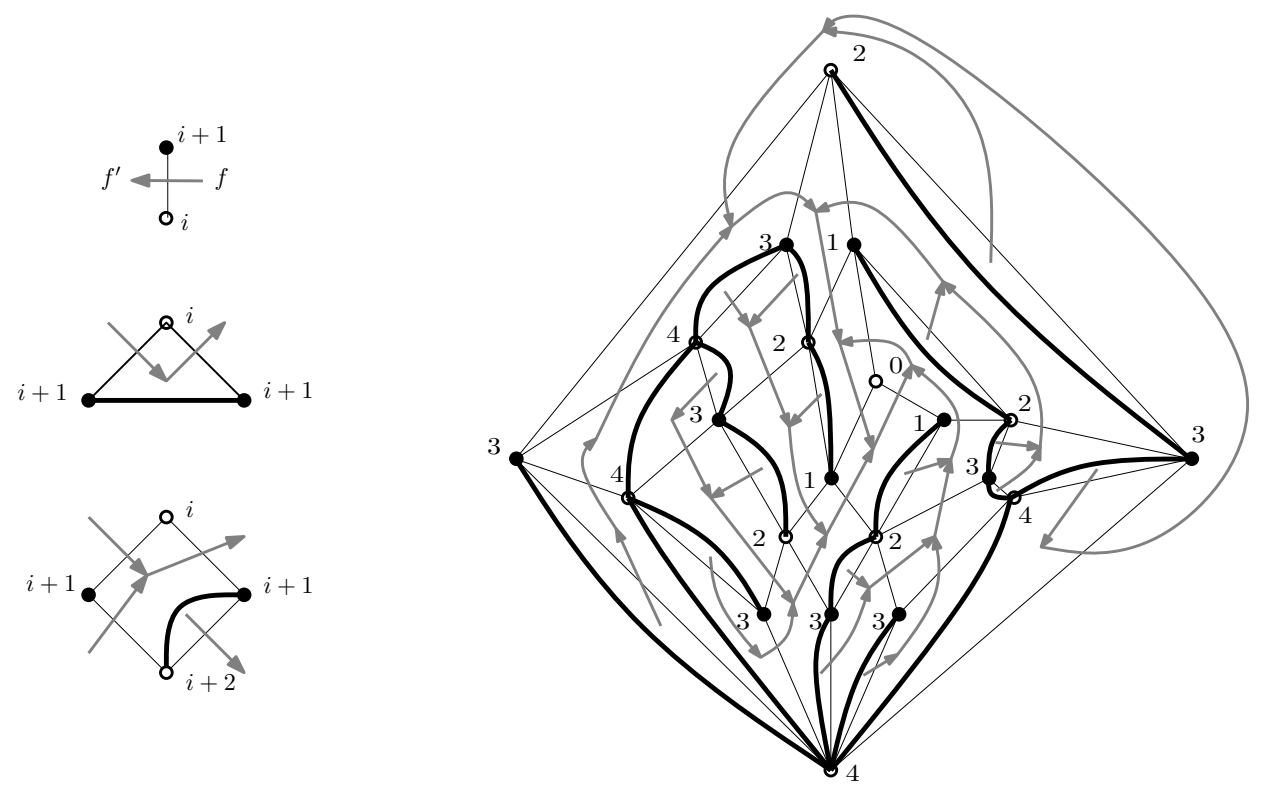

Fig. 5. The orientation of dual old edges in $\mathfrak{q}^{\prime}$ : local configurations and an example.

Let us prove that the fake edges form a forest of oriented trees attached to a unique oriented cycle around $v_{0}$. Consider the 3 types of faces of $\mathfrak{q}^{\prime}$ as represented on Figure 5: There is exactly one outgoing fake edge in each face, that is, from each fake vertex, and all oriented fake edges are weakly decreasing. Hence an oriented cycle of fake edges connects fake vertices with equal labels. But such a cycle turns counterclockwise around a unique vertex $v$ of $\mathfrak{q}$, which must be $v_{0}$ since all its neighbors have larger labels.

To conclude the proof of the lemma, observe that there can be only one face in $\phi\left(\mathfrak{q}, v_{0}\right)$ since the fake edges connect all faces of $\mathfrak{q}^{\prime}$, and that any loop in this face can be retracted along the tree like structure of fake edges.

\subsection{Well labelled polygons, chords diagrams, and the closure of a well labelled $g$-tree}

We now describe a mapping $\psi$ which we later claim to be the inverse of $\phi$. The construction of $\psi(\mathfrak{t})$ will take place in the unique face of $\mathfrak{t}$, which is homeomorphic to a disk by definition of maps: in particular the actual genus of $S_{g}$ will be somewhat irrelevant for this reverse construction, which will reveal to be a simple adaptation of the construction used in [Sch98; CS04; MM06] for the planar $(g=0)$ case. More precisely, given a labelled $g$-tree $\mathfrak{t}$ with $n$ edges, we decompose it into a rooted $g$-tree $\overrightarrow{\mathfrak{t}}_{0}$ (forgetting the labels) and a well labelled rooted $2 n$-gon $\overrightarrow{\mathfrak{p}}$ (describing the sequence of labels along the border of the unique face of $\mathfrak{t}$ ).

We will use the following folklore combinatorial result:

LEMmA 3. Let $\overrightarrow{\mathfrak{p}}$ be a rooted labelled $m$-gon with labels $a_{1}, \ldots, a_{m}$ in clockwise order starting after the root edge, and assume that the labels satisfy the Lukasiewicz condition $a_{i+1}-a_{i} \geq-1$, and $a_{i}>a_{m}$ for all $i$. 

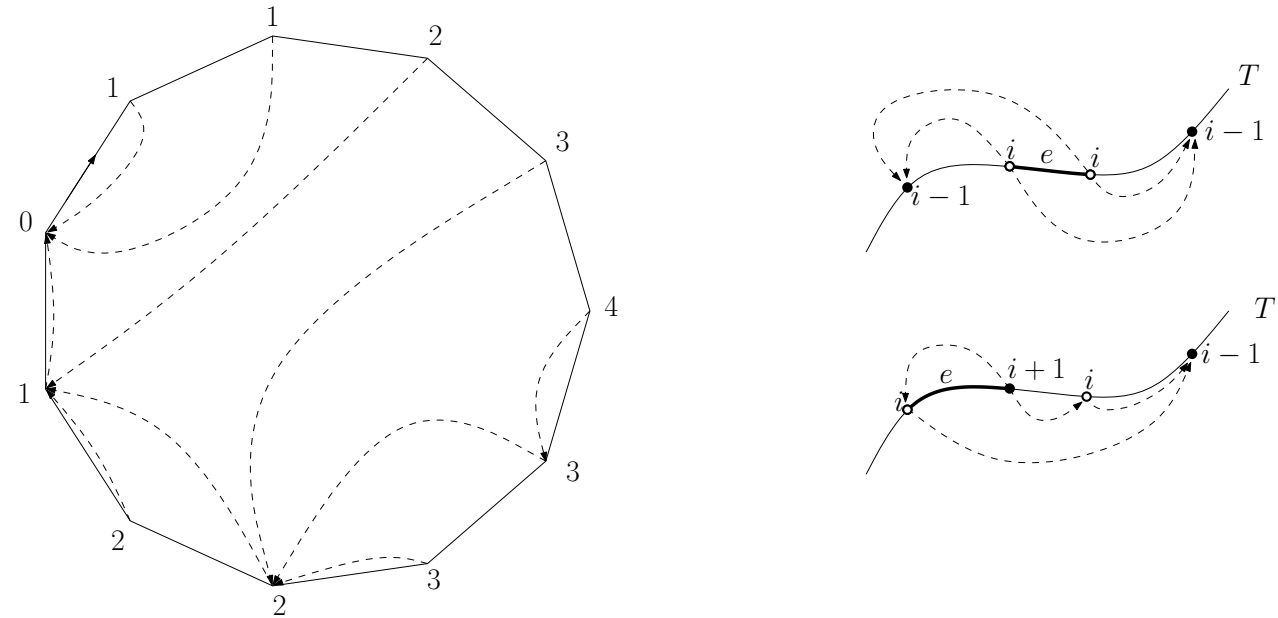

Fig. 6. The dissection of a polygon and the two types of faces of $\psi(\mathfrak{t})$

Let $\pi:\{1, \ldots, m-1\} \rightarrow\{2, \ldots, m\}$ be the application that maps $i$ to its predecessor, that is the smallest $j>i$ such that $a_{j}=a_{i}-1$.

Then there exists a unique way to draw all the edges $(i, \pi(i))$ inside $\overrightarrow{\mathfrak{p}}$ so that the resulting graph drawing is a planar map. In this map, each inner face is incident exactly once to the polygon, along an edge $\left(a_{i}, a_{i+1}\right)$, and the labels on the border of this face are then, in clockwise direction, $\left(a_{i}, a_{i+1}, a_{i+1}-1, a_{i+1}-2, \ldots, a_{i+1}-\delta\right)$ where $\delta=a_{i+1}-a_{i}+1$ (in particular $\delta \geq 0$ and the face incident to $\left(a_{i}, a_{i+1}\right)$ has degree $\delta+2)$.

Proof. See [Sta99], or the detailed discussion of the appendix.

The closure of a well labeled $g$-tree $\mathfrak{t}$ with $n$ edges is the graph drawing $\psi(\mathfrak{t})$ constructed as follows: Let $\mathfrak{p}$ be the labelled $2 n$-gon describing the face $f$ of $\mathfrak{t}$, and let $k$ be the number of vertices with label 1 in $\mathfrak{p}$ : these vertices corresponds to corners with label 1 of $f$. Let $\mathfrak{t}^{\prime}$ be the map obtained by adding a vertex $v_{0}$ with label 0 in $f$ and $k$ edges that join this vertex to each of the corners with label 1 of $f$ : the map $\mathfrak{t}^{\prime}$ has $k$ faces and the border of each face is a polygon $\overrightarrow{\mathfrak{p}}_{i}$ with minimal label 0 (taken as root) and labels that satisfies the Lukasiewicz condition (the variations are even in $\{+1,0,-1\}$ ). In each face of the map $\mathfrak{t}^{\prime}$ (on $\mathcal{S}_{g}$ ), that is in each polygon $\overrightarrow{\mathfrak{p}}_{i}$, the above lemma can thus be used to add all the edges between corners with positive labels and their predecessor, in the unique way that provides a map $\mathfrak{t}^{\prime \prime}$ on $\mathcal{S}_{g}$. The closure $\psi(\mathfrak{t})$ of $\mathfrak{t}$ is the graph drawing $\mathfrak{t}^{\prime \prime}-\mathfrak{t}$ on $\mathcal{S}_{g}$ obtained by removing the edges of $\mathfrak{t}$ from $\mathfrak{t}^{\prime \prime}$.

LEMMA 4. The graph drawing $\psi(\mathfrak{t})$ is a quadrangulation of genus $g$ with $n$ edges.

Proof. According to Lemma 3 each face of $\mathfrak{t}^{\prime}$ is incident exactly once to an edge of $\mathfrak{t}$. Each edge $e$ of $\mathfrak{t}$ thus separates two different faces, which are otherwise incident only to edges of $\mathfrak{t}^{\prime}-\mathfrak{t}$. Moreover since the variation of labels are opposite $\pm \delta$ on the two sides of $e$, the removal of $e$ creates a simply connected face of degree $\delta+2+(-\delta+2)-2=4$, as illustrated by Figure 6 . The removal of the $n$ edges of $\mathfrak{t}$ thus creates $n$ quadrangular faces. 


\subsection{The main theorem}

The main combinatorial result of this paper is the following theorem, that states that not only $\phi\left(\mathfrak{q}, v_{0}\right)$ is a well labelled map on $\mathcal{S}_{g}$ but the mapping $\phi$ is more precisely a bijection.

THEOREM 1. The opening $\phi$ is a bijection between

- pointed bipartite quadrangulations of genus $g$ that have $n$ faces, $2 n$ edges, and $n+2-2 g$ vertices ( $i$ of which are at odd distance of the basepoint), and

- well labeled $g$-trees that have $n$ edges, and $n+1-2 g$ vertices ( $i$ of them having odd labels),

such that the labels of the $g$-tree associated to a bipartite quadrangulation $\mathfrak{q}$ with basepoint $v_{0}$ give the distances of vertices of $\mathfrak{q}$ to $v_{0}$.

Proof. The fact that $\phi(\psi(\mathfrak{t}))=\mathfrak{t}$ follows from the comparison of Figure 6 and Figure 4: for the two types of faces created in $\psi(\mathfrak{t})$, the edge $e$ is indeed recovered correctly by the opening rules. The fact that $\psi\left(\phi\left(\mathfrak{q}, v_{0}\right)\right)=\left(\mathfrak{q}, v_{0}\right)$ follows from the fact that in $\mathfrak{q}^{\prime}=\mathfrak{q} \cup \phi\left(\mathfrak{q}, v_{0}\right)$, each edge of $\mathfrak{q}$ joins a corner of $\phi\left(\mathfrak{q}, v_{0}\right)$ to its predecessor, and from the unicity in Lemma 3. An alternative direct proof is proposed in the appendix.

In the enumerative context one usually prefers to deal with rooted maps to avoid the discussion of symmetries. The opening is readily extended to rooted bipartite quadrangulations: define the image of a rooted quadrangulation $(\mathfrak{q}, \vec{e})$ with root vertex $v_{0}$, to be $\left(\phi\left(\mathfrak{q}, v_{0}\right), \overrightarrow{e^{\prime}}\right)$ where $e^{\prime}$ is the edge created in the root face of $(\mathfrak{q}, \vec{e})$, oriented so that its origin is the endpoint of $\vec{e}$ (observe that, according to the rules and the definition of the root face, the endpoint of $\vec{e}$ is indeed incident to $e^{\prime}$ ).

Corollary 1. The opening $\phi$ extends to a bijection between

- rooted bipartite quadrangulations with genus $g$ that have $n$ faces, and

- rooted well labeled $g$-trees that have $n$ edges.

Proof. By construction the origin of $\overrightarrow{e^{\prime}}$ has label 1 so that $\left(\phi\left(\mathfrak{q}, v_{0}\right), \overrightarrow{e^{\prime}}\right)$ is indeed a rooted well labeled tree. Conversely given a rooted well labeled tree $\overrightarrow{\mathfrak{t}}=\left(\mathfrak{t}, \overrightarrow{e^{\prime}}\right)$ with root vertex $v$, taking as root edge of $\psi(\overrightarrow{\mathfrak{t}})$ the edge $\vec{e}$ of $\mathfrak{t}^{\prime}$ with origin $v_{0}$ which follows $\overrightarrow{e^{\prime}}$ in counterclockwise direction around $v$ immediately yields the unique possible preimage of $\overrightarrow{\mathfrak{t}}$.

It turns out that for our purpose an even better variant consists in taking a basepoint and a root independently. Define the image of a rooted pointed bipartite quadrangulation $\left(\mathfrak{q}, \vec{e}, v_{0}\right)$ to be $\left(\phi\left(\mathfrak{q}, v_{0}\right), \overrightarrow{e^{\prime}}, s\right)$ where $\overrightarrow{e^{\prime}}$ is the new edge created in the root face of $\mathfrak{q}$, oriented so that its origin is the extremity of $\vec{e}$ with largest label, and where $s \in\{+1,-1\}$ is a sign recording the orientation of $\vec{e}$ (say + if $\vec{e}$ is increasing with respect to the labeling). Let moreover $\nu$ be the relabeling mapping that simultaneously translates all labels of an embedded map so that the root vertex label is 1 . Then

Corollary 2. Opening followed by relabelling is a bijection $\nu \circ \phi$ between - rooted pointed bipartite quadrangulations with genus $g$ that have $n$ faces, and 
— pairs consisting of a rooted embedded $g$-tree that has $n$ edges and a sign \pm 1 .

Proof. In view of Theorem $1, \phi$ can be used to map bijectively rooted pointed bipartite quadrangulations $\left(\mathfrak{q}, v_{0}, \vec{e}\right)$ of genus $g$ with $n$ faces onto almost well labelled rooted g-trees $\left(\phi\left(\mathfrak{q}, v_{0}\right), \vec{e}\right)$ with $n$ edges (almost well labeled means the minimum label is 1 but the root vertex has not necessarily label 1). Now $\nu$ is bijective between almost well labelled $g$-trees with $n$ edges and embedded rooted $g$-trees with $n$ edges: indeed given a rooted embedded map (with root vertex label 1 by definition), there is a unique way to translate its labels so that the minimum becomes 1 .

The point of this last corollary is that, as we shall see in Section 4, embedded $g$-trees are much easier to count than well labeled $g$-trees.

\section{THE ENUMERATION OF QUADRANGULATIONS VIA SCHEMES}

In view of Theorem 1 and Corollary 2, the enumeration of maps of genus $g$ according to the number of vertices, faces and edges can be reduced to the enumeration of rooted well labeled or embedded $g$-trees. It turns out that rooted embedded $g$ trees are easier to deal with, so that we shall rely on the following transposition of Corollary 2 in terms of generating series:

COROLlary 3. The generating series

$$
\overrightarrow{Q_{g}} \cdot(z)=\sum_{n \geq 2 g} \vec{q}_{g, n}^{\bullet} z^{n}, \quad \text { and } \quad T_{g}(z)=\sum_{n \geq 2 g} a_{g, n} z^{n}
$$

of rooted pointed bipartite quadrangulations of genus $g$ (with respect to the number of faces) and of rooted embedded g-trees (with respect to the number of edges) are related by the following equation:

$$
\overrightarrow{Q_{g}}(z)=2 T_{g}(z)
$$

Since a quadrangulation with genus $g$ and $n$ faces has $n+2-2 g$ vertices, $\vec{q}_{g, n}^{\bullet}=$ $(n+2-2 g) \vec{q}_{g, n}$, and the previous equation can also be rewriten in terms of the generating series of rooted bipartite quadrangulations of genus $g$ as

$$
Q_{g}(z)=\sum_{n \geq 2 g} \vec{q}_{g, n} z^{n}=z^{2 g-2} \int \overrightarrow{Q_{g}}(y) y^{1-2 g} d y
$$

with the initial condition $Q_{g}(z)=O\left(z^{2 g}\right)$.

\subsection{Planar maps and embedded trees}

Let $T \equiv T(z)=T_{0}(z)$ be the generating series of rooted embedded trees with respect to the number of edges. A rooted embedded tree $\overrightarrow{\mathfrak{t}}$ which has at least 1 edge can be decomposed into two rooted trees $\overrightarrow{\mathfrak{t}}_{1}$ and $\overrightarrow{\mathfrak{t}}_{2}$ by deleting its root edge $e$. The subtree $\overrightarrow{\mathfrak{t}}_{1}$ rooted at the origin of $e$ has root label 1 , so that it is again a rooted embedded tree. The subtree $\overrightarrow{\mathfrak{t}}_{2}$ rooted at the endpoint of $e$ has root label $\delta \in\{+1,0,-1\}$ but, up to a translation of labels by $1-\delta$, it is also a rooted embedded tree. This decomposition is a bijection $\overrightarrow{\mathfrak{t}} \mapsto\left(\delta, \overrightarrow{\mathfrak{t}}_{1}, \overrightarrow{\mathfrak{t}}_{2}\right)$ between non empty rooted embedded trees with $n$ edges and triples consisting of an edge variation and 
two rooted embedded trees with a total of $n-1$ edges. Hence the equation

$$
T(z)=\sum_{\overrightarrow{\mathfrak{t}}} z^{|\overrightarrow{\mathfrak{t}}|}=1+3 \sum_{\left(\overrightarrow{\mathfrak{t}}_{1}, \overrightarrow{\mathfrak{t}}_{2}\right)} z^{1+\left|\overrightarrow{\mathfrak{t}}_{1}\right|+\left|\overrightarrow{\mathfrak{t}}_{2}\right|}=1+3 z T(z)^{2} .
$$

The unique formal power series root of this quadratic equation $T=1+3 z T^{2}$ is

$$
T(z)=\frac{1-\sqrt{1-12 z}}{6 z} .
$$

In particular $a_{n}=\left[z^{n}\right] T(z)=\frac{3^{n}}{n+1}\left(\begin{array}{c}2 n \\ n\end{array}\right)$ is the number of embedded trees with $n$ edges. For future reference, let us also observe the relations

$$
z=\frac{T-1}{3 T^{2}}, \quad z T^{\prime}=\frac{T^{2}-T}{2-T}, \quad \text { and } \quad 1+\frac{2 z T^{\prime}}{T}=\frac{T}{2-T} .
$$

The enumeration of planar quadrangulations then directly follows from Corollary 3.

COROLLARY 4. The generating series of planar rooted pointed quadrangulations satisfies

$$
{\overrightarrow{Q_{0}}}_{\bullet}^{\bullet}(z)=2 T(z)
$$

and the number of planar rooted quadrangulations with $n$ faces is

$$
\vec{q}_{0, n}=\frac{\vec{q}_{0, n}^{\bullet}}{n+2}=\frac{2}{n+2} \cdot \frac{3^{n}}{n+1}\left(\begin{array}{c}
2 n \\
n
\end{array}\right) .
$$

\subsection{Reduced $g$-trees and tree grafting}

We say that a $g$-tree is reduced if it has no vertices of degree one. Upon deleting vertices of degree 1 iteratively, a reduced $g$-tree can be extracted from any $g$-tree.

LEMмa 5. There is a bijection between rooted embedded $g$-trees with $n$ edges and pairs consisting of a rooted embedded reduced $g$-tree $\overrightarrow{\mathfrak{r}}$ with $k$ edges and a forest $\left(\overrightarrow{\mathfrak{t}}_{1}, \ldots, \overrightarrow{\mathfrak{t}}_{2 k}\right)$, where,

$-\overrightarrow{\mathfrak{t}}_{2}, \ldots, \overrightarrow{\mathfrak{t}}_{2 k}$ are rooted embedded planar trees.

$-\overrightarrow{\mathfrak{t}}_{1}$ is either a rooted or a doubly rooted embedded planar tree,

and the total number of edges in $\left(\overrightarrow{\mathfrak{r}} ; \overrightarrow{\mathfrak{t}}_{1}, \ldots, \overrightarrow{\mathfrak{t}}_{2 k}\right)$ is $n$.

ProOF. Given a $g$-tree $\mathfrak{t}$, a unique reduced $g$-tree $\mathfrak{r}$ is obtained by iteratively deleting in $\mathfrak{t}$ the vertices of degree 1 until none is left. At the end of this process, the deleted edges form a forest of rooted embedded planar trees: one (possibly trivial) rooted tree is attached to each corner of $\mathfrak{r}$ (see Figure 7 ). The root $\vec{e}$ of $\overrightarrow{\mathfrak{t}}$, then provides a root for $\overrightarrow{\mathfrak{r}}$ : if $\vec{e}$ was not deleted it becomes the root of $\overrightarrow{\mathfrak{r}}$, otherwise $\vec{e}$ belongs to one of the deleted trees $\overrightarrow{\mathfrak{t}}_{1}$ and the root of $\overrightarrow{\mathfrak{r}}$ is taken to be the arc of $\mathfrak{r}$ on the right hand side of which $\overrightarrow{\mathfrak{t}}_{1}$ is attached.

Once $\overrightarrow{\mathfrak{r}}$ is rooted, its corners are canonically ordered starting with the corner on the right hand side of the root, and the deleted trees can be arranged into an ordered list: the $g$-tree $\mathfrak{t}$ can then be recovered from $\overrightarrow{\mathfrak{r}}$ and the ordered list $\left(\overrightarrow{\mathfrak{t}}_{1}, \ldots, \overrightarrow{\mathfrak{t}}_{2 k}\right)$ of trees. Finally, when the root of $\overrightarrow{\mathfrak{t}}$ is not an edge of $\overrightarrow{\mathfrak{r}}$ it must be recorded as a secondary root of $\overrightarrow{\mathfrak{t}}_{1}$. 


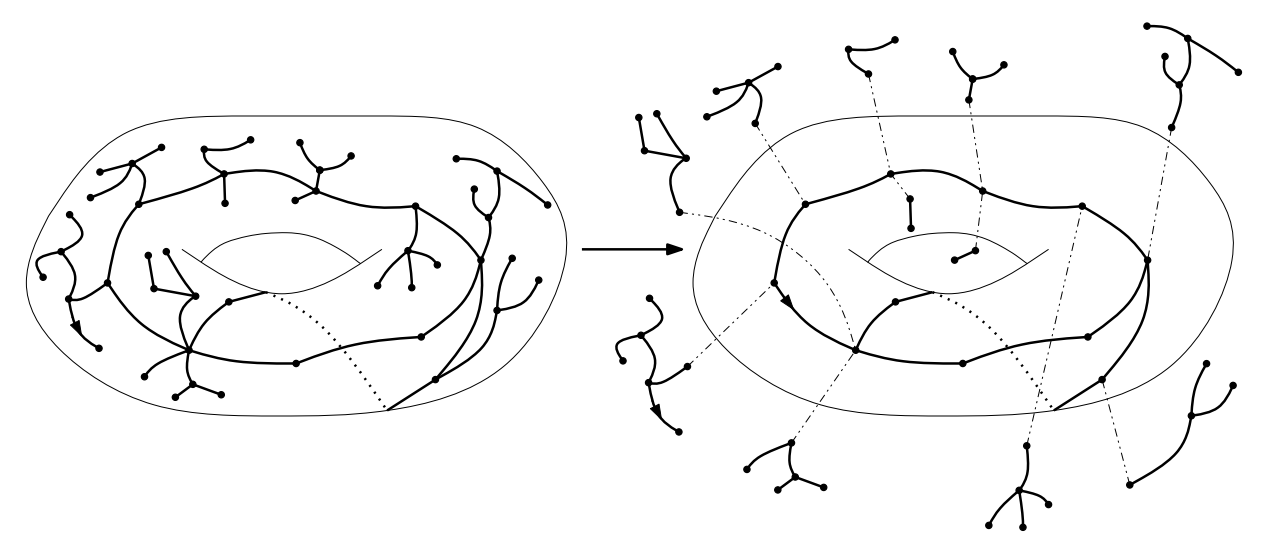

Fig. 7. A 1-tree and its decomposition into a reduced 1-tree and a collection of rooted planar trees attached on its corners (only planar trees which are not reduced to a single vertex are represented).

Lemma 5 immediately yields the following corollary.

COROLlaRY 5. The generating series of rooted embedded $g$-trees $T_{g}$ and of rooted embedded reduced $g$-trees $R_{g}$ are related by:

$$
T_{g}(z)=R_{g}\left(z T(z)^{2}\right) \cdot\left(1+\frac{2 z T^{\prime}(z)}{T(z)}\right)=R_{g}\left(z T^{2}\right) \cdot \frac{T}{2-T} .
$$

In terms of the primitive $\widehat{R}_{g}(t)$, satisfying $\frac{t \partial}{\partial t} \widehat{R}_{g}(t)=R_{g}(t)$, the previous formula reads:

$$
T_{g}(z)=\frac{z \partial}{\partial z}\left(\widehat{R}_{g}\left(z T^{2}\right)\right)
$$

Proof. The decomposition of a rooted $g$-tree $\mathfrak{t}$ in a pair $\left(\overrightarrow{\mathfrak{r}} ; \overrightarrow{\mathfrak{t}}_{1}, \ldots, \overrightarrow{\mathfrak{t}}_{2 k}\right)$ where $k$ is the number of edges of $k$ is a combinatorial composition operation that results in the composition of generating series:

$$
\begin{aligned}
T_{g}(z) & =\sum_{\overrightarrow{\mathfrak{t}}} z^{|\overrightarrow{\mathfrak{t}}|}=\sum_{\overrightarrow{\mathfrak{r}} ; \overrightarrow{\mathfrak{t}}_{1}, \overrightarrow{\mathfrak{t}}_{2} \ldots, \overrightarrow{\mathfrak{t}}_{2 k}|k=| \overrightarrow{\mathfrak{r}} \mid} z^{|\overrightarrow{\mathfrak{r}}|+\left|\overrightarrow{\mathfrak{t}}_{1}\right|+\left|\overrightarrow{\mathfrak{t}}_{2}\right|+\ldots+\left|\overrightarrow{\mathfrak{t}}_{2 k}\right|} \\
& =\sum_{\overrightarrow{\mathfrak{r}}} z^{|\overrightarrow{\mathfrak{r}}|}\left(\sum_{\overrightarrow{\mathfrak{t}}}(1+2 \mid \overrightarrow{\mathfrak{t}}) z^{|\overrightarrow{\mathfrak{t}}|}\right)\left(\sum_{\overrightarrow{\mathfrak{t}}} z^{|\overrightarrow{\mathfrak{t}}|}\right)^{2|\overrightarrow{\mathfrak{r}}|-1}=R_{g}\left(z T(z)^{2}\right) \cdot\left(1+\frac{2 z T^{\prime}}{T}\right),
\end{aligned}
$$

where the notation $\overrightarrow{\vec{t}}_{1}$ stresses the fact that the first tree can be doubly rooted: given a tree $\mathfrak{t}$ there are $2|\mathfrak{t}|$ ways to introduce a double root and one way not to. The multiplicative factor $1+2 z T^{\prime}(z) / T(z)$ can also be understood directly, saying that the first rooted tree (with generating series $T(z)$ ) can be replaced by a doubly rooted tree (with generating series $2 z T^{\prime}(z)$ ).

The second expression can be checked by a direct comparison with the first formula:

$$
\frac{z \partial}{\partial z}\left(\widehat{R}_{g}\left(z T^{2}\right)\right)=z\left(T^{2}+2 z T T^{\prime}\right) \widehat{R}_{g}^{\prime}\left(z T^{2}\right)
$$




$$
=\left(z T^{2}\right) \widehat{R}_{g}^{\prime}\left(z T^{2}\right)\left(1+2 z T^{\prime} / T\right)=R_{g}\left(z T^{2}\right) \cdot\left(1+\frac{2 z T^{\prime}}{T}\right) .
$$

By definition the series $\widehat{R}_{g}$ is a generating series of rooted maps in which rooted maps with $e$ edges are counted with a weight $1 / e$ : it is tempting to think about it as a generating series of unrooted maps, but then each unrooted map is counted with a weight proportional to the inverse of the size of its automorphism group (because maps with non trivial automorphisms have less than $2 e$ distinct rootings). In these terms the second expression of Corollary 5 could be understood directly as follows: rooted $g$-trees are first constructed as unrooted by grafting trees in an unrooted reduced $g$-tree, and then rooted. However a rigourous proof along these lines requires a careful discussion of symmetries, which is circumvented by working directly with rooted objects.

\subsection{The standard scheme of a reduced $g$-tree}

A labeled scheme is a rooted $g$-tree without vertices of degree 1 and 2 that has integer labels on vertices. A scheme is standard if its labels form an integer interval with minimum 0 . Observe that the labels of a scheme are not required to vary at most by one along edges, and that in a standard scheme all the integers of the interval must appear as labels.

Let $\overrightarrow{\mathfrak{r}}$ be a rooted reduced $g$-tree. By definition the map $\overrightarrow{\mathfrak{r}}$ has no vertices of degree 1. Its vertices of degree 2 are organized into maximal paths, connected together at vertices of degree at least 3 . Let $\overrightarrow{\mathfrak{s}}_{0}$ and $\overrightarrow{\mathfrak{s}}$ be the maps obtained from $\overrightarrow{\mathfrak{r}}$ as follows:

-To get $\overrightarrow{\mathfrak{s}}_{0}$ from $\overrightarrow{\mathfrak{r}}$, replace each maximal path made of vertices of degree 2 by an edge, the path containing the root of $\overrightarrow{\mathfrak{r}}$ providing a rooted edge for $\overrightarrow{\mathfrak{s}}_{0}$.

- To get $\overrightarrow{\mathfrak{s}}$ from $\overrightarrow{\mathfrak{s}}_{0}$, standardize the labels of the vertices, so that they form an integer interval with minimum label 0: if the labels appearing on vertices of degree at least 3 in $\overrightarrow{\mathfrak{r}}$ are $\ell_{0}<\ell_{1}<\ldots<\ell_{p}$ then the corresponding labels in $\overrightarrow{\mathfrak{s}}$ are $0,1, \ldots, p$ (a given label can appear on several vertices of $\overrightarrow{\mathfrak{s}}_{0}$, so that $p$ may be smaller than the number of vertices of $\overrightarrow{\mathfrak{s}}$.).

The maps $\overrightarrow{\mathfrak{s}}_{0}$ and $\overrightarrow{\mathfrak{s}}$ are labeled schemes, and $\overrightarrow{\mathfrak{s}}$ is standard: it is called the standard scheme of $\overrightarrow{\mathfrak{r}}$. By extension, $\overrightarrow{\mathfrak{s}}$ is called the standard scheme of $\overrightarrow{\mathfrak{q}}$ if $\overrightarrow{\mathfrak{r}}$ was itself extracted from the $g$-tree $\overrightarrow{\mathfrak{t}}$ associated to a quadrangulation $\overrightarrow{\mathfrak{q}}$. Finally, given a rooted standard scheme $\overrightarrow{\mathfrak{s}}$ with labels $\{0, \ldots, p\}$ and $p$ positive integers $\ell_{1}<\ldots<\ell_{p}$ let $\overrightarrow{\mathfrak{s}}\left(\ell_{1}, \ldots, \ell_{p}\right)$ be the rooted labeled scheme obtained by replacing labels $i$ by $\ell_{i}$ in $\overrightarrow{\mathfrak{s}}$.

Observe that on every maximal degree 2 path of $\overrightarrow{\mathfrak{r}}$, the labels perform a sequence of small variations taken from $\{+1,0,-1\}$. A Motzkin walk $w$ of length $\ell$ is defined as a finite sequence $w_{1} \ldots w_{\ell}$ with $w_{i} \in\{+1,0,-1\}$. The quantity $\sum_{i=0}^{\ell} w_{i}$ is called the increment of $w$. A Motzkin walk of increment 0 is called a Motzkin bridge. In these terms, on every maximal path of $\mathfrak{r}$, the sequence of variations of labels forms a Motzkin walk: hence, if the standard scheme $\overrightarrow{\mathfrak{s}}$ of $\overrightarrow{\mathfrak{r}}$ has $k$ edges, a $k$-uple of Motzkin walks $\left(\mathfrak{m}_{1}, \ldots, \mathfrak{m}_{k}\right)$ can be extracted from $\overrightarrow{\mathfrak{r}}$, with $\mathfrak{m}_{i}$ the Motzkin walk read along the path of $\overrightarrow{\mathfrak{r}}$ corresponding to $i$ th edge of $\overrightarrow{\mathfrak{s}}$ in the canonical order.

A $k$-uple of Motzkin walks is said to be compatible with a rooted standard scheme with $k$ edges if there exists an embedded rooted reduced $g$-tree from which they 


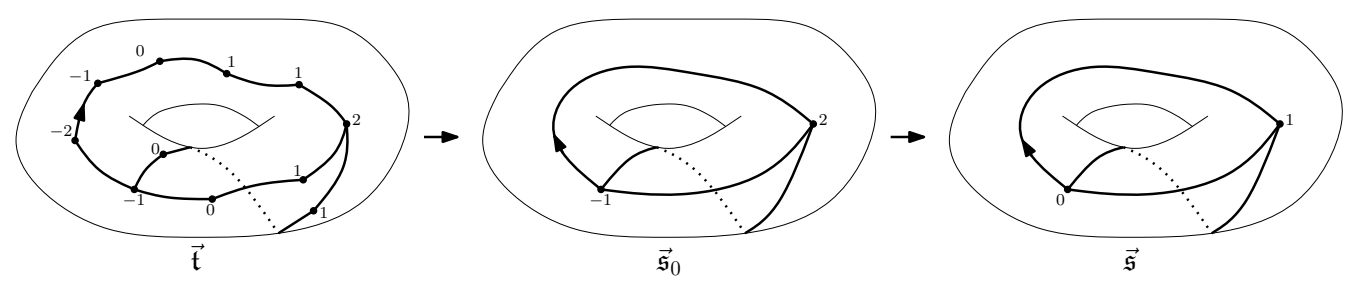

Fig. 8. A reduced 1-tree and its rooted scheme.

can be both extracted. Observe that the $g$-tree is unique up to translation of all labels, since it is recovered by replacing the $i$ th edge of the scheme by a chain of vertices with variations given by the $i$ th Motzkin walk.

Lemma 6. There is a bijection between embedded rooted reduced $g$-trees with $n$ edges such that the root vertex has degree at least 3, and pairs formed of a rooted standard scheme of genus $g$ with $k$ edges, and a compatible $k$-uple of non empty walks with a total of $n$ steps.

Proof. In view of the definition of compatible $k$-uples of walks, it suffices to show that if the pair $\left(\overrightarrow{\mathfrak{s}} ;\left(\mathfrak{m}_{1}, \ldots, \mathfrak{m}_{k}\right)\right)$ is extracted from embedded rooted reduced $g$-tree $\mathfrak{t}$ then $\mathfrak{t}$ can be recovered from the pair: this is immediate upon replacing the $i$ th edge of $\overrightarrow{\mathfrak{s}}$ by a path with labels given by $\mathfrak{m}_{i}$, and choosing the root to be the first edge of the first path.

In particular, given a rooted standard scheme $\overrightarrow{\mathfrak{s}}$ and a compatible $k$-uple of Motzkin walks $\left(\mathfrak{m}_{1}, \ldots, \mathfrak{m}_{k}\right)$ let $\vec{r}\left(\overrightarrow{\mathfrak{s}} ; \mathfrak{m}_{1}, \ldots, \mathfrak{m}_{k}\right)$ be the unique corresponding rooted reduced $g$-tree, and $\overrightarrow{\mathfrak{s}}_{0}\left(\overrightarrow{\mathfrak{s}} ; \mathfrak{m}_{1}, \ldots, \mathfrak{m}_{k}\right)$ be the rooted labeled scheme extracted from $\vec{r}\left(\overrightarrow{\mathfrak{s}} ; \mathfrak{m}_{1}, \ldots, \mathfrak{m}_{k}\right)$ before standardization.

LEMMA 7. Let $\overrightarrow{\mathfrak{s}}$ be a rooted standard scheme with $k$ edges and labels $\{0,1, \ldots, p\}$, and let $\ell_{1}<\ldots<\ell_{p}$ be positive integers.

Then the set of compatible $k$-uples such that $\overrightarrow{\mathfrak{s}} 0\left(\overrightarrow{\mathfrak{s}} ; \mathfrak{m}_{1}, \ldots, \mathfrak{m}_{k}\right)=\overrightarrow{\mathfrak{s}}\left(\ell_{1}, \ldots, \ell_{k}\right)$ is exactly the set of $k$-uples $\left(\mathfrak{m}_{1}, \ldots, \mathfrak{m}_{k}\right)$ such that, for all $i, \mathfrak{m}_{i}$ has increment $\ell_{e_{i}^{+}}-\ell_{e_{i}^{-}}$, where $e_{i}^{-}$and $e_{i}^{+}$are the labels of the origin and endpoint of the ith edge $\overrightarrow{e_{i}}$ of $\overrightarrow{\mathfrak{s}}$.

Proof. If a $k$-uple is compatible with $\overrightarrow{\mathfrak{s}}$ and induces $\ell_{0} \ldots \ell_{p}$ then the labels of the corresponding reduced $g$-tree satisfy the conditions of the lemma. Conversely, given $\ell_{0}, \ldots, \ell_{p}$, a corresponding embedded reduced $g$-tree is obtained by replacing, for $i=1, \ldots, k$, the $i$ th edge $\vec{e}_{i}$ of $\overrightarrow{\mathfrak{s}}$ by a labelled path constructed as follows: if $\mathfrak{m}_{i}=w_{1} \ldots w_{\ell}$ and $e_{i}^{-}$is the label of the origin of $\vec{e}_{i}$ then the inserted path has length $\ell$ and its $j$ th vertex has label $\ell_{e}+\sum_{m=1}^{j} w_{m}$, for $j=0, \ldots, \ell$. The condition on increments ensures that each vertices of $\overrightarrow{\mathfrak{s}}$ get the same label from all its incident edges. A root can be chosen arbitrarily on the first path and labels simultaneously translated to obtain an embedded rooted reduced $g$-tree.

Let $\overrightarrow{\mathfrak{s}}$ be a rooted standard scheme with $v$ vertices, $k$ edges and labels $\{0, \ldots, p\}$. 
We define the weight of $\overrightarrow{\mathfrak{s}}$ as the following power series:

$$
W_{\overrightarrow{\mathfrak{s}}}(t)=\frac{1}{k} \sum_{0<\ell_{1}<\ldots<\ell_{p}} \prod_{i=1}^{k} M_{\left|\ell_{e_{i}^{+}}-\ell_{e_{i}^{-}}\right|}(t),
$$

where $e_{i}^{-}$and $e_{i}^{+}$denote the label of the extremities of the $i$ th edge $\vec{e}_{i}$ of $\overrightarrow{\mathfrak{s}}$, and, for all $j \geq 0, M_{j}(t)$ is the generating series of non-empty Motzkin walks of increment $j$ with respect to the length: $M_{j}(t)=\sum_{\mathfrak{m}} t^{|\mathfrak{m}|}$, where the sum is over all Motzkin walks with increment $j$ and length at least 1 .

LEMMA 8. The primitive $\widehat{R_{g}}(t)$ of the generating series of embedded rooted reduced $g$-trees satisfies

$$
\widehat{R_{g}}(t)=\sum_{\overrightarrow{\mathfrak{s}}} W_{\overrightarrow{\mathfrak{s}}}(t)
$$

where the sum is taken over all rooted standard schemes of genus $g$.

ProOF. Let us group together in a series $R_{g, k}(t)$ the contributions to the generating series $R_{g}(t)$ of the embedded reduced $g$-trees whose standard scheme has $k$ edges, so that $R_{g}(t)=\sum_{k} R_{g, k}(t)$. Then $2 k R_{g, k}(t)$ is the generating series of rooted embedded reduced $g$-trees whose standard scheme has $k$ edges and carries a secondary root-edge leaving a vertex of degree at least 3 : indeed, each maximal path of a given reduced $g$-tree provides 2 choices of such a secondary root.

Now, these rooted embedded reduced $g$-trees having a secondary root can also be obtained by considering first a rooted reduced $g$-tree with a root vertex of degree at least 3 (and whose standard scheme has $k$ edges), and then choosing a root edge. Hence we have:

$$
2 k R_{g, k}(t)=2 \frac{t d}{d t} \sum_{|\overrightarrow{\mathfrak{s}}|=k} \widetilde{R}_{\overrightarrow{\mathfrak{s}}}(t)
$$

where the sum is taken over rooted standard schemes of genus $g$ with $k$ edges, and where $\widetilde{R}_{\overrightarrow{\mathfrak{s}}}(t)$ is the generating series of embedded reduced $g$-trees of scheme $\overrightarrow{\mathfrak{s}}$ with a root vertex of degree at least 3 (the derivative accounts for the choice of the root edge, and the factor 2 for the choice of its orientation).

Now, in view of Lemmas $6-7$, for each scheme $\overrightarrow{\mathfrak{s}}$ with $k$ edges, the generating series $\widetilde{R}_{\overrightarrow{\mathfrak{s}}}(t)$ is obtained by summing over all relabellings and compatible $k$-uples of Motzkin paths. Therefore:

$$
\widetilde{R}_{\overrightarrow{\mathfrak{s}}}(t)=\sum_{0<\ell_{1}<\ldots<\ell_{p}} \prod_{i=1}^{k} M_{\left|\ell_{e_{i}^{+}}-\ell_{e_{i}^{-}}\right|}(t) .
$$

Putting Equations 5 and 6 together and using the definition of $W_{\vec{s}}$ gives:

$$
R_{g, k}(t)=\frac{t d}{d t} \sum_{|\overrightarrow{\mathfrak{s}}|=k} W_{\overrightarrow{\mathfrak{s}}}(t)
$$

Summing on $k$ and comparing with the definition of $\widehat{R_{g}}(t)$ yields the result. 


\subsection{The algebra of Motzkin walks}

A Motzkin walk is primitive if it has increment -1 but none of its prefix has increment -1 . In other terms a Motzkin walk $w=w_{1} \ldots w_{\ell}$ is primitive if it satisfies the Eukasiewicz property: $\sum_{i=1}^{\ell} w_{i}=-1$ but for all $j<\ell, \sum_{i=1}^{\ell} w_{i} \geq 0$.

Let $U \equiv U(t)=\sum_{w} t^{|w|}$ be the generating series of primitive Moztkin walks according to the length, and $B \equiv B(t)=M_{0}(t)$ be the generating series of nonempty bridges. Using a decomposition at the first step for $U$, and at the first return to the $x$-axis for $B$, one gets:

$$
\begin{aligned}
& U=t\left(1+U+U^{2}\right) \\
& B=t(1+2 U)(1+B)=\frac{t(1+2 U)}{1-t(1+2 U)} .
\end{aligned}
$$

Then the explicit value of $U$ and $B$ are

$$
U=\frac{1}{2 t}\left(1-t-\left(\left(1-2 t-3 t^{2}\right)^{1 / 2}\right), \quad \text { and } \quad 1+B=\frac{1}{\left(1-2 t-3 t^{2}\right)^{1 / 2}} .\right.
$$

In terms of $U$, the series $B$ satisfies

$$
B=\frac{U(1+2 U)}{1-U^{2}}, \text { and } 1+B=\frac{1+U+U^{2}}{1-U^{2}} .
$$

Decomposing walks at their last passage time at each positive integer, one obtains the generating function of Motzkin walks of increment $i>0$ :

$$
M_{i}(t)=(1+B) U^{i}
$$

The algebra of Laurent power series generated by the series $U$ contains $t$ since

$$
t=\frac{U}{1+U+U^{2}}=\frac{1}{U^{-1}+1+U} .
$$

More generally we would like to characterize rational expressions in $U$ that are in fact rational expressions in $t$. Obviously these expressions are symmetric in the exchange $U \leftrightarrow U^{-1}$. This is also a sufficient condition since the polynomials $\left(U+1+U^{-1}\right)^{k}$ form a base of the set of polynomials in $\left\{U, U^{-1}\right\}$ that are symmetric in these two indeterminates.

Lemma 9. A rational series in $U$ is a rational series in $t$ if and only if it is symmetric in the exchange $U \leftrightarrow U^{-1}$.

\subsection{The torus}

Let $\mathfrak{r}$ be a reduced 1-tree with $k$ edges, and let $d_{i}$ be the number of vertices of degree $i$ in $\mathfrak{r}$. Then the vertex-edge incidence relation and Euler's formula give the following 2 relations:

$$
\left\{\begin{aligned}
2 k & =\sum_{i \geq 1} i d_{i}, \\
k & =1+\sum_{i \geq 1} d_{i} .
\end{aligned}\right.
$$

Eliminating $k$ and recording that $d_{1}=0$ since $\mathfrak{r}$ is reduced, this yields

$$
\sum_{i \geq 2}(i-2) d_{i}=2 \text {. }
$$


Hence, apart from vertices of degree 2, there can be only one vertex of degree 4 $\left(d_{3}=0, d_{4}=1\right)$ or two vertices of degree $3\left(d_{3}=2, d_{4}=0\right)$. Let us compute the standard schemes and weights associated to these two types of reduced 1-trees:

1 vertex of degree 4. If the 1 -tree consists of 1 vertex of degree 4 and vertices of degree 2, its standard scheme has just 1 vertex of degree 4 and 2 edges that are loops. There is a unique rooted 1-tree $\overrightarrow{\mathfrak{t}}_{1}$ with one vertex of degree four, and giving label 0 to its unique vertex we obtain the unique rooted standard scheme $\overrightarrow{\mathfrak{s}}_{1}$ with 1 vertex. The associated weight is therefore

$$
W_{\overrightarrow{\mathfrak{s}}_{1}}=\frac{1}{2} M_{0}^{2}=\frac{1}{2} B^{2}=\frac{1}{2} \frac{(1+2 U)^{2} U^{2}}{(1-U)^{2}(1+U)^{2}} .
$$

2 vertices of degree 3. If instead the 1-tree consists of two vertices of degree 3 and vertices of degree 2 , its standard scheme has two vertices of degree 3 and 3 edges connecting them. There is again a unique rooted 1-tree with 2 vertices of degree 3 but the choice of a label $a$ for the root vertex and $b$ for the second vertex yields three different rooted standard schemes $\overrightarrow{\mathfrak{s}}_{2}, \overrightarrow{\mathfrak{s}}_{3}, \overrightarrow{\mathfrak{s}}_{4}$ :

$-a=b=0$ : in this case the endpoints of all three edges have label 0 , so that the associated weight is

$$
W_{\overrightarrow{\mathfrak{s}}_{2}}=\frac{1}{3} B^{3}=\frac{1}{3} \frac{(1+2 U)^{3} U^{3}}{(1-U)^{3}(1+U)^{3}} .
$$

$-a=0, b=1:$ in this case the associated weight is

$$
W_{\overrightarrow{\mathfrak{s}}_{3}}=\frac{1}{3} \sum_{\ell_{1}>0}\left((1+B) U^{\ell_{1}}\right)^{3}=\frac{1}{3}(1+B)^{3} \frac{U^{3}}{1-U^{3}}=\frac{2}{3} \frac{\left(1+U+U^{2}\right)^{2} U^{3}}{(1-U)^{4}(1+U)^{3}}
$$

$-a=1, b=0$ : this case yields the same weight as the previous one, $W_{\overrightarrow{\mathfrak{s}}_{4}}=W_{\overrightarrow{\mathfrak{s}}_{3}}$.

Summing over the different cases, we get

$$
\begin{aligned}
\widehat{R_{1}} & =W_{\overrightarrow{\mathfrak{s}}_{1}}+W_{\overrightarrow{\mathfrak{s}}_{2}}+W_{\overrightarrow{\mathfrak{s}}_{3}}+W_{\overrightarrow{\mathfrak{s}}_{4}} \\
& =\frac{U^{-1}+4+U}{2\left((1-U)\left(1-U^{-1}\right)\right)^{2}(1+U)\left(1+U^{-1}\right)} .
\end{aligned}
$$

We thus obtained $\widehat{R_{1}}$ as a rational function of $U$. Its symmetry w.r.t. the exchange $U \leftrightarrow U^{-1}$ shows that it is in fact a rational function of $t$ : indeed one easily checks that

$$
\widehat{R}_{1}=\frac{t^{2}(1+3 t)}{2(1-3 t)^{2}(1+t)}
$$

In view of Corollary 5 we obtain the generating function of rooted 1-trees as

$$
T_{1}(z)=\frac{z \partial}{\partial z} \widehat{R}_{1}\left(z T(z)^{2}\right)
$$

and deduce from Corollary 3 an expression for the generating series of pointed rooted bipartite quadrangulation on the torus as ${\overrightarrow{Q_{g}}}^{\bullet}(z)=2 T_{1}(z)$. However, in view of Formula 3, one can derive directly the generating series of rooted bipartite 
quadrangulations on the torus from $\widehat{R}_{1}$ :

$$
\begin{aligned}
Q_{1}(z) & =\int \frac{d y}{y} \overrightarrow{Q_{1}} \cdot(y)=2 \int \frac{d y}{y} \frac{y d}{d y} \widehat{R}_{1}\left(y T(y)^{2}\right) \\
& =2 \widehat{R}_{1}\left(z T(z)^{2}\right)=2 \widehat{R}_{1}\left(\frac{T(z)-1}{3}\right)=\frac{(T-1)^{2} T}{3(2-T)^{2}(2+T)},
\end{aligned}
$$

in agreement with the result of [BC91].

\subsection{General surfaces}

In general the incidence and Euler relations for reduced $g$-trees with $k$ edges and $d_{i}$ vertices of degree $i$ are

$$
\left\{\begin{aligned}
2 k & =\sum_{i \geq 1} i d_{i} \\
k & =2 g-1+\sum_{i \geq 1} d_{i} .
\end{aligned}\right.
$$

Eliminating $k$ and using $d_{1}=0$, this yields

$$
\sum_{i \geq 2}(i-2) d_{i}=4 g-2
$$

There is thus again a finite number of possible combinations for the $d_{i}$ with $i \geq 3$ : in particular the two extremal possibilities in terms of number of edges are:

$-d_{4 g}=1$, and $d_{i}=0$ otherwise, which leads to schemes with 1 vertex of degree $4 g$ and $2 g$ edges, the minimal number of edges for a scheme of genus $g$.

$-d_{3}=4 g-2$, and $d_{i}=0$ otherwise, which leads to schemes with $4 g-2$ vertices of degree 3 and $6 g-3$ edges, the maximal number of edges for a scheme of genus $g$.

Let $\overrightarrow{\mathfrak{s}}$ be a rooted standard scheme with $q$ vertices and $k$ edges $(k=2 g-1+q)$, Let us call $p$ the number of distinct nonzero labels in $\overrightarrow{\mathfrak{s}}$ (in particular $0 \leq p \leq q$ ), $\mathfrak{e}$ the set of edges with equal extremities, and $\mathfrak{e}^{\prime}$ its complement. Then we have by definition:

$$
W_{\overrightarrow{\mathfrak{s}}}=\frac{1}{k} B^{|\mathfrak{e}|} \sum_{0<\ell_{1}<\ldots<\ell_{p}} \prod_{e \in \mathfrak{e}^{\prime}} M_{\left|\ell_{e_{-}}-\ell_{e_{+}}\right|},
$$

where $e_{-}$and $e_{+}$denote the vertex labels of the extremities of the edge $e$ of $\mathfrak{e}^{\prime}$.

Observe that for $0 \leq i<i^{\prime} \leq v_{\neq}$, we have $\ell_{i}<\ell_{i^{\prime}}$ and

$$
M_{\ell_{i^{\prime}}-\ell_{i}}=(1+B) U^{\ell_{i^{\prime}}-\ell_{i}}=(1+B) \prod_{j=i+1}^{i^{\prime}} U^{\ell_{j}-\ell_{j-1}},
$$

so that

$$
W_{\overrightarrow{\mathfrak{s}}}=\frac{1}{k} B^{|\mathfrak{e}|}(1+B)^{\left|\mathfrak{e}^{\prime}\right|} \sum_{0<\ell_{1}<\ldots<\ell_{p}} \prod_{e \in \mathfrak{e}^{\prime}} \prod_{j=e_{-+1}}^{e_{+}} U^{\ell_{j}-\ell_{j-1}} .
$$

Since the expression summand only involves the differences $\delta_{j}=\ell_{j}-\ell_{j-1}$, the sum can be rewritten in terms of these $p$ new variables:

$$
W_{\overrightarrow{\mathfrak{s}}}=\frac{1}{k} B^{|\mathfrak{e}|}(1+B)^{\left|\mathfrak{e}^{\prime}\right|} \sum_{\delta_{1}, \ldots, \delta_{p}} \prod_{e \in \mathfrak{e}^{\prime}} \prod_{j=e_{-+1}}^{e_{+}} U^{\delta_{j}},
$$


where the $\delta_{j}$ are independant summation indices over positive integers. Now for each couple $(j, e)$ such that $e_{-}<j \leq e_{+}$a contribution $U^{\delta_{j}}$ is obtained. Let $d(j)$ denote the number of edges $e$ such that the couple $(j, e)$ satisfies the previous condition. Observe that the $d(j)$ only depend on $\overrightarrow{\mathfrak{s}}$, not on the $\delta_{j}$. Then

$$
\begin{aligned}
W_{\overrightarrow{\mathfrak{s}}} & =\frac{1}{k} B^{|\mathfrak{e}|}(1+B)^{\left|\mathfrak{e}^{\prime}\right|} \sum_{\delta_{1}, \ldots, \delta_{v_{p}}} \prod_{j=1}^{p} U^{d(j) \delta_{j}} \\
& =\frac{1}{k} B^{|\mathfrak{e}|}(1+B)^{\left|\mathfrak{e}^{\prime}\right|} \prod_{j=1}^{p} \frac{U^{d(j)}}{1-U^{d(j)}} .
\end{aligned}
$$

Setting $e_{=}=|\mathfrak{e}|$ and $e_{\neq}=\left|\mathfrak{e}^{\prime}\right|$, and expressing $B$ in terms of $U$ we obtain

$$
W_{\overrightarrow{\mathfrak{s}}}=\frac{1}{k} \frac{U^{e=}(1+2 U)^{e}=\left(1+U+U^{2}\right)^{e} \neq}{\left(1-U^{2}\right)^{k}} \prod_{j=1}^{p} \frac{U^{d(j)}}{1-U^{d(j)}} .
$$

In view of Lemma 8 , the generating series of reduced labeled $g$-trees is obtained upon summing these individual contributions for all rooted standard schemes of genus $g$.

THEOREM 2. Let $\mathcal{W}_{g}$ denote the (finite) set of rooted standard schemes of genus g. Given such a standard scheme $\overrightarrow{\mathfrak{s}}$ with $k$ edges and labels $\{0,1, \ldots, p\}$, let $e_{=}$ denote the number of edges with equal labels at both ends, and $e_{\neq}=k-e_{=}$. For $j=1, \ldots, p$, denote moreover by $d(j)$ the number of edges $e$ with endpoint labels satisfying $e_{-}<j \leq e_{+}$, and $d=\sum_{j=1}^{p} d(j)$. Then

$$
\widehat{R}_{g}(t)=\sum_{\overrightarrow{\mathfrak{s}} \in \mathcal{W}_{g}} \frac{1}{k} \frac{U^{d+e}=(1+2 U)^{e}=\left(1+U+U^{2}\right)^{e} \neq}{(1-U)^{k+p}(1+U)^{k}} \prod_{j=1}^{p} \frac{1}{1+U+\ldots+U^{d(j)-1}}(7)
$$

where $U$ is the formal power series in $t$ satisfying $U=t\left(1+U+U^{2}\right)$. Moreover

$$
T_{g}(z)=\frac{z \partial}{\partial z} \widehat{R}_{g}\left(z T^{2}\right), \quad{\overrightarrow{Q_{g}}}_{\bullet}(z)=2 T_{g}(z)
$$

where $T$ is the formal power series in $z$ satisfying $T=1+3 z T^{2}$.

In particular we expressed $\overrightarrow{Q_{g}}(z)$ as a rational function of $U\left(z T^{2}\right)$, which is an algebraic function of degree 4 . It is proved however in [BC91] that $Q_{g}(z)$ is in fact a rational function in $z$ and $\sqrt{1-12 z}$. One can verify that this result implies that our series $\widehat{R}_{g}(t)$ is in fact a rational function in $t$, or in other terms that the expression of $\widehat{R}_{g}(t)$ in terms of $U$ is in fact symmetric in the exchange $U \leftrightarrow U^{-1}$. We are currently unable to give a combinatorial proof of this fact.

\subsection{Asymptotics}

In Equation (7), there are a finite number of critical values of $U$, all being roots of unity, and $U=1$ is one of them. As a fuction of $t, U$ reaches the value $U=1$ at its singularity $t=1 / 3$. Moreover, $U$ being a power series in $t$ with positive coefficients, one has $|U(t)| \leq 1$ for every complex number $t$ with $|t| \leq 1 / 3$, with equality only at $t=1 / 3$. This implies that $t=1 / 3$ is the only dominant singularity 
of $R_{g}(t)$. Finally, $U(t)$ is an algebraic function, which admits a Puiseux expansion at its singularity:

$$
U=1-\sqrt{3} \sqrt{1-3 t}+O(1-3 t) .
$$

This implies the following corollary:

COROLlary 6 . The dominant singularity of the algebraic function $\widehat{R}_{g}(t)$ is at $t=1 / 3$, around which the following Puiseux expansion holds:

$$
\widehat{R}_{g}(t)=\sum_{\overrightarrow{\mathfrak{s}} \in \mathcal{W}_{g}} \frac{1}{k}\left(\frac{3}{2}\right)^{k}\left(\frac{1}{\sqrt{3} \sqrt{1-3 t}}\right)^{k+p} \prod_{j=1}^{p} \frac{1}{d(j)} \cdot(1+O(\sqrt{1-3 t})),
$$

where for each $\overrightarrow{\mathfrak{s}}$ in the summation, $k$ is the number of edges and $\{0,1, \ldots, p\}$ the set of distinct labels.

The dominant terms in this sum are given by the standard schemes $\overrightarrow{\mathfrak{s}}$ with $k+p$ maximal: $p$ is at most $q-1$ if $\overrightarrow{\mathfrak{s}}$ has $q$ vertices and all labels are distinct. Then $k+q-1=2 q+2 g-2$, which is maximal for $q=d_{3}=4 g-2$, resulting in $k+q-1=10 g-6$.

COROLLARY 7. Let $\mathcal{W}_{g}^{m}$ denote the set of rooted standard schemes of genus $g$ with $4 g-2$ vertices of degree 3 that are labelled by distinct integers. Then

$$
\widehat{R}_{g}(t)=\frac{1}{6 g-3} \frac{3^{g}}{2^{6 g-3}}\left(\sum_{\vec{s} \in \mathcal{W}_{g}^{m}} \prod_{i=1}^{4 g-3} \frac{1}{d(i)}\right)\left(\frac{1}{1-3 t}\right)^{5 g-3} \cdot(1+O(\sqrt{1-3 t})) .
$$

In order to obtain the asymptotic behavior of $\overrightarrow{Q_{g}}(z)$, we need to consider the behavior of $T$ : this algebraic series has a unique dominant singularity at $z=1 / 12$ and

$$
T=2-2 \sqrt{1-12 z}+O(1-12 z)
$$

so that

$$
z T^{2}=\frac{T-1}{3}=\frac{1}{3}-\frac{2}{3} \sqrt{1-12 z}+O(1-12 z) .
$$

Since $T$ is a power series in $z$ with positive coefficients, one has $\left|z T^{2}\right|<1 / 3$ for every $z \neq 1 / 12$ such that $|z| \leq 1 / 12$, so that we can compose the singular expansions of $T$ and $\widehat{R}_{g}$ in the expression $\overrightarrow{Q_{g}}(z)=\frac{z d}{d z} \widehat{R}_{g}\left(z T^{2}\right)$ of Corollary 3 to obtain:

COROLlaRY 8. The algebraic series $\overrightarrow{Q_{g}} \cdot(z)$ has a unique dominant singularity at $z=1 / 12$, around which the following Puiseux expansion holds:

$\overrightarrow{Q_{g}} \cdot(z)=\frac{5 g-3}{6 g-3} \frac{3^{g}}{2^{11 g-6}}\left(\sum_{\overrightarrow{\mathfrak{s}} \in \mathcal{W}_{g}^{m}} \prod_{i=1}^{4 g-3} \frac{1}{d(i)}\right)\left(\frac{1}{\sqrt{1-12 z}}\right)^{5 g-1} \cdot\left(1+O\left((1-12 z)^{1 / 4}\right)\right.$

It is a well known fact that algebraic functions are amenable to singularity analysis (see [FO90; FS08]). In other words, classical transfer theorems apply to the previous expansion, leading to: 
COROLlary 9. The number $\vec{q}_{g, n}^{\bullet}$ of rooted pointed bipartite quadrangulations of genus $g$ with $n$ faces satisfies:

$$
\vec{q}_{g, n}^{\bullet} \sim \frac{1}{\Gamma\left(\frac{5 g-1}{2}\right)} \frac{5 g-3}{6 g-3} \frac{3^{g}}{2^{11 g-6}}\left(\sum_{\overrightarrow{\mathfrak{s}} \in \mathcal{W}_{g}^{m}} \prod_{i=1}^{4 g-3} \frac{1}{d(i)}\right) \cdot n^{\frac{5 g-3}{2}} \cdot 12^{n} .
$$

Recalling that $q_{g, n}^{\bullet}=(n+2-2 g) \vec{q}_{g, n}^{\bullet}$ and using the product relation $\Gamma\left(\frac{5 g-1}{2}\right)=$ $\frac{5 g-3}{2} \Gamma\left(\frac{5 g-3}{2}\right)$, we finally have:

COROLLARY 10. The number of rooted bipartite quadrangulations of genus $g$ with $n$ faces satisfies

$$
q_{g, n} \sim \frac{3^{g}}{(6 g-3) 2^{11 g-7} \Gamma\left(\frac{5 g-3}{2}\right)}\left(\sum_{\overrightarrow{\mathfrak{s}} \in \mathcal{W}_{g}^{m}} \prod_{i=1}^{4 g-3} \frac{1}{d(i)}\right) \cdot n^{\frac{5}{2}(g-1)} \cdot 12^{n} .
$$

Observe that the maps in $\mathcal{W}_{g}^{m}$ are vertex labeled rooted maps with $4 g-2$ vertices of degree 3 , one face and degree $g$. Their number is $(4 g-2) ! \varepsilon_{g}$ where $\varepsilon_{g}$ is the number of cubic maps of genus $g$ with one face (or dually triangulations with one vertex), known to be [WL72]:

$$
\varepsilon_{g}=\frac{2}{12^{g}} \frac{(6 g-3) !}{g !(3 g-2) !} .
$$

In particular this allows to give bounds on the constant $\sum_{\overrightarrow{\mathfrak{s}} \in \mathcal{W}_{g}^{m}} \prod_{i} \frac{1}{d(i)}$. For fixed $g$, this constant is in principle a computable finite sum. However, in practice, since the number of maps in $\mathcal{W}_{g}^{m}$ grows superexponentially in $g$, the computation is difficult to perform in a reasonable time. In particular we were able to obtain in this way the explicit value of the constants only for $g=1,2$, leading to the following asymptotics, in agreement with [BC91]:

$$
q_{1, n} \sim \frac{1}{24} \cdot 12^{n} \text { and } q_{2, n} \sim \frac{7}{4320 \sqrt{\pi}} \cdot n^{5 / 2} \cdot 12^{n} .
$$

Several different explicit non linear recursions are known for these constants (see [BC91; DGZJ95; Wit91]), but we were unable to derive them from our expressions.

\section{CONCLUSION}

As in the planar case, our main bijection maps the distances to the basepoint of a quadrangulation onto the labels of vertices of the associated $g$-tree. In particular it allows to prove that the distances of a random vertex to the basepoint in a uniform random quadrangulation with $n$ faces is again of order $n^{1 / 4}$ : a detailed analysis in this direction was recently done in [Mie07] for the Bolzmann distribution on quadrangulations of genus $g$. A natural problem, partially considered there, is more generally to construct a continuum limit of large quadrangulations in this $n^{1 / 4}$ scaling: in view of the results of [LG07; LGP07] in the planar case, a continuum limit of quadrangulations of genus $g$ is expected to have the topology of $\mathcal{S}_{g}$.

We conjecture that the resulting continuum random quadrangulation of genus $g$ would be universal enough to describe the continuum limit of all models of uniform 
random maps with simple face degree or connectivity constraints, but also the continuum limit of uniform random graphs with $n$ vertices and minimum genus $g$.

As we have seen the dominant terms in the asymptotic of the number of maps on a surface of genus $g$ arises from maps with schemes that have the maximum number of vertices and edges. More precisely with probability going to 1 as $n$ goes to infinity, a random map of genus $g$ has a scheme with $4 g-2$ vertices of degree 3 .

The asymptotic behavior appears to have an interesting structure. As shown in [Cha] the more general construction of Bouttier et al [BDFG04] in terms of labeled mobiles extends to higher genus in a similar way as our original quadrangular construction. As a result one obtains a more general class of labeled $g$-trees, say $g$-mobiles. The decomposition of [Cha] is more involved but leads to the same dominant standard schemes, with weights involving, instead of $U$ and $T$, a finite number of algebraic series depending on the set of allowed degrees. In particular the same constant

$$
\tau_{g}=\sum_{\vec{s} \in \mathcal{W}_{g}^{m}} \prod_{i=1}^{4 g-3} \frac{1}{d(i)}
$$

drives the multiplicative constant in the asymptotic behavior, in agreement with the pattern that was observed by [Gao93], and with the universality described in the physics literature [DGZJ95].

\section{ACKNOWLEDGMENTS}

This paper is based in part on early unpublished works of the last two authors [Mar97; Sch98] in their PhD theses under the direction of Robert Cori. We wish here to express our warm thanks to Robert Cori for his interest and support for this project. We also thank Gregory Miermont for communicating us [Mie07] and for interesting discussions.

\section{REFERENCES}

Jan Ambjø rn, Bergfinnur Durhuus, and Thordur Jónsson. Quantum gravity, a statistical field theory approach. Cambridge Monographs on Mathematical Physics, 1997.

Edward A. Bender and E. Rodney Canfield. The number of rooted maps on an orientable surface. J. Combin. Theory Ser. B, 53(2):293-299, 1991.

Jérémie. Bouttier, Philippe Di Francesco, and Emmanuel Guitter. Planar maps as labeled mobiles. Electron. J. Combin., 11(1):Research Paper 69, 27 pp. (electronic), 2004.

Edward Bender. Some unsolved problems in map enumeration. Bull. Inst. Combin. Appl., 3:51-56, 1991.

Edouard Brézin, Claude Itzykson, Giorgio Parisi, and Jean-Bernard Zuber. Planar diagrams. Comm. Math. Phys., 59(1):35-51, 1978.

Guillaume Chapuy. On the asymptotic number of constellations on orientable surfaces. manuscript, in preparation.

Philippe Chassaing and Gilles Schaeffer. Random planar lattices and integrated superBrownian excursion. Probab. Theory Related Fields, 128(2):161-212, 2004.

Robert Cori and Bernard Vauquelin. Planar maps are well labeled trees. Canad. J. Math., 33(5):1023-1042, 1981.

Philippe Di Francesco, Paul Ginsparg, and Jean Zinn-Justin. 2d gravity and random matrices. Physics Reports, 254:1, 1995. 
Philippe Flajolet and Andrew Odlyzko. Singularity analysis of generating functions. SIAM J. Discrete Math., 3(2):216-240, 1990.

Philippe Flajolet and Robert Sedgewick. Analytic Combinatorics. preliminary version avalaible on the web : http://algo.inria.fr/flajolet/Publications, 2008.

Zhicheng Gao. The number of degree restricted maps on general surfaces. Discrete Math., 123(13):47-63, 1993.

Jean-François Le Gall. The topological structure of scaling limits of large planar maps. Inventiones Mathematica, 169:621-670, 2007.

Jean-François Le Gall and Frédéric Paulin. Scaling limits of bipartite planar maps are homeomorphic to the 2-sphere. preprint (see www.dma.ens.fr/legall/), 2007.

Michel Marcus. Cartes, hypercartes, et diagrammes de cordes. PhD thesis, Université Bordeaux $1,1997$.

Grégory Miermont. Tessellations of random maps of arbitrary genus. manuscript, 60pp, december, 2007.

Jean-François Marckert and Abdelkader Mokkadem. Limit of normalized quadrangulations: the Brownian map. Ann. Probab., 34(6):2144-2202, 2006.

Bojan Mohar and Carsten Thomassen. Graphs on surfaces. The Johns Hopkins University Press, Baltimore and London, 2001.

Gilles Schaeffer. Conjugaison d'arbres et cartes combinatoires aléatoires. PhD thesis, Université Bordeaux 1, 1998.

Richard Stanley. Enumerative Combinatorics, volume II. Cambdridge Series in Advanced Mathematics, 1999.

William T. Tutte. A census of planar maps. Canad. J. Math., 15:249-271, 1963.

Edward Witten. Two-dimensional gravity and intersection theory on moduli space. Surveys Diff. Geom., 1:243-310, 1991.

Timothy R. S. Walsh and Alfred B. Lehman. Counting rooted maps by genus. I. J. Combinatorial Theory Ser. B, 13:192-218, 1972.

Edward M. Wright. The number of connected sparsely edged graph. J. Graph Theory, 1(317-330), 1977.

Edward M. Wright. The number of connected sparsely edged graph. ii. smooth graphs and blocks. J. Graph Theory, 2(299-305), 1978.

\section{A. ELEMENTARY MAP SURGERY}

\section{A.1 Corners, edge addition and deletion}

Let $v$ be a vertex of degree $k$ of a map $\mathfrak{m}$. The $k$ arcs with origin $v$ in $\mathfrak{m}$ form a cycle around $v$ and the corner $c_{a a^{\prime}}$ between two successive $\operatorname{arcs} a$ and $a^{\prime}$ in counterclockwise order around $v$ is the sector of a small neighborhood of $v$ between these arcs. The corner $c_{a a^{\prime}}$ is said to be incident to $v$ and to the face which is on the left hand side of $a$ (and on the right hand side of $a^{\prime}$ ). A corner $c$ is incident to a unique vertex $v$ and a unique face $f$, so we sometime simply say that $c$ is a corner of $v$ or a corner of $f$.

Given a map $\mathfrak{m}$ and two (possibly identical) corners $c_{1}$ and $c_{2}$ incident to a same face $f$, there is a unique way to create a map $\mathfrak{m}^{\prime}$ by adding inside $f$ an edge $e$ that connects the vertices incident to $c_{1}$ and $c_{2}$ at these corners: by this operation the face $f$ of $\mathfrak{m}$ is naturally divided in $\mathfrak{m}^{\prime}$ into two faces $f^{\prime}$ and $f^{\prime \prime}$ separated by the edge $e$, and the corners $c_{1}$ and $c_{2}$ are respectively divided into $c_{1}^{\prime}$ and $c_{1}^{\prime \prime}$ and $c_{2}^{\prime}$ and $c_{2}^{\prime \prime}$ accordingly. Observe that if $\mathfrak{m}$ is a map of genus $g$ with $n$ edges, $v$ vertices and $f$ faces, then $\mathfrak{m}^{\prime}$ is a map of genus $g$ with $n+1$ edges, $v$ vertices and $f+1$ faces, in accordance with Euler's formula. 
Given a map $\mathfrak{m}$ and a subset $\mathfrak{e}$ of its edge set, we now consider the problem of deleting $\mathfrak{e}$ from $\mathfrak{m}$. Let us denote by $\mathfrak{m}-\mathfrak{e}$ the graph drawing obtained by ignoring the edges of $\mathfrak{e}$ in the map $\mathfrak{m}$. A priori, $\mathfrak{m}-\mathfrak{e}$ can only be considered as a graph drawing because the removal of some edges from $\mathfrak{m}$ can disconnect the underlying graph or more generally it can create non simply connected faces. The following lemma gives sufficent conditions on $\mathfrak{e}$ that garanty that $\mathfrak{m}-\mathfrak{e}$ is a map. From a topological point of view, it is an elementary application of Van Kampen theorem.

LEMMA 10. Let $f_{1}$ and $f_{2}$ be 2 adjacent faces of a map $\mathfrak{m}$ on $\mathcal{S}_{g}$, and e an edge separating $f_{1}$ and $f_{2}$. Then $\mathfrak{m}-\{e\}$ is a map on $\mathcal{S}_{g}$.

Let $v_{0}$ be a vertex of degree $d$ of a map $\mathfrak{m}$ on $\mathcal{S}_{g}$ such that $v_{0}$ is incident to d different faces, and let $e_{1}, \ldots, e_{d}$ be the edges incident to $v_{0}$. Then $\mathfrak{m}-\left\{v_{0}, e_{1}, \ldots, e_{d}\right\}$ is a map on $\mathcal{S}_{g}$.

Proof. For the first alinea, Van Kampen theorem can be applied directly: the union of two simply connected regions with a simply connected intersection is simply connected. The second alinea follows by iteration.

Implicitely in the previous discussion and in the rest of the text we use the fact that the deletion or adjunction of edges and vertices to a graph drawing that preserve the surface and the map structure commute to the homeomorphisms of the surface, so that these operations make sense up to homeomorphisms. As already said, the interested reader can refer to [MT01] for a purely combinatorial treatment of these questions.

\section{A.2 Duality and spanning trees}

Given a map $\mathfrak{m}$, the dual map is obtained by exchanging the role of vertices and faces and keeping incidence relations: more precisely to construct the dual map $\mathfrak{m}^{*}$ of $\mathfrak{m}$, put a vertex in each face of $\mathfrak{m}$ and connect these vertices by a dual edge $e^{*}$ across each edge of $\mathfrak{m}$. Given a map $\mathfrak{m}$ and a set of edges $\mathfrak{e}$ of $\mathfrak{m}$, let $\mathfrak{e}^{*}$ denote the subset $\left\{e^{*} \mid e \in \mathfrak{e}\right\}$ of the set of edges of the dual map $\mathfrak{m}^{*}$. In terms of dual edges, the first alinea of Lemma 10 admits an immediate extension:

LEMma 11. Let $\mathfrak{e}$ be a set of edges of a map $\mathfrak{m}$ such that $\mathfrak{e}^{*}$ is a forest (that is, the edges of $\mathfrak{e}^{*}$ do not form any cycle in $\left.\mathfrak{m}^{*}\right)$. Then $\mathfrak{m}-\mathfrak{e}$ is a map on $\mathcal{S}_{g}$.

Proof. Let $e \in \mathfrak{e}$. We wish to apply Lemma 10 to show that $m-\{e\}$ is a map and conclude by induction since $(\mathfrak{e} \backslash\{e\})^{*}$ is a forest with less edges. The only problem could be that $e$ is incident twice to the same face $f$ : but this would mean, in dual terms, that $e^{*}$ is incident twice to the same vertex, a contradiction with the assumption that the edges of $\mathfrak{e}^{*}$ form no cycle.

We shall use the following immediate corollary of the previous lemma.

Corollary 11. If $\mathfrak{e}^{*}$ is a spanning tree of $\mathfrak{m}^{*}$ (that is, the edges of $\mathfrak{e}^{*}$ do not form any cycle, and any two vertices of $\mathfrak{m}^{*}$ can be joined by a path made of edges of $\left.\mathfrak{e}^{*}\right)$, then $\mathfrak{m}-\mathfrak{e}$ is a $g$-tree.

Proof. By Lemma $11, \mathfrak{m}-\mathfrak{e}$ is a map on $\mathcal{S}_{g}$. Let $f_{1}$ and $f_{2}$ be two faces of $\mathfrak{m}-\mathfrak{e}$. Each of them contains at least one vertex of $\mathfrak{m}^{*}$, and since $\mathfrak{e}^{*}$ is a spanning tree these two vertices are connected by a path made of edges of $\mathfrak{e}^{*}$. By definition of 
dual edges this path does not cross the graph $\mathfrak{m}-\mathfrak{e}$ : it stays in the same connected component of $\mathcal{S}_{g} \backslash(\mathfrak{m}-\mathfrak{e})$, so that $f_{1}=f_{2}$.

Observe that in the planar case $g=0$, this is the classical duality result: if $\mathfrak{e}^{*}$ spans $\mathfrak{m}^{*}$ then $\mathfrak{m}-\mathfrak{e}$ spans $\mathfrak{m}$.

\section{B. THE CORRECTNESS OF THE MAIN BIJECTION}

\section{B.1 The mapping $\phi$ produces a well defined map}

Our first aim is to prove Lemma 2: the graph drawing created by the opening $\phi$ is a map on $\mathcal{S}_{g}$ with one face. This will essentially be done by applying Lemma 11.

Let $\left(\mathfrak{q}, v_{0}\right)$ be a pointed bipartite quadrangulation of genus $g$ with $n$ faces, and let $\mathfrak{q}^{\prime}=\mathfrak{q} \cup \phi\left(\mathfrak{q}, v_{0}\right): \mathfrak{q}^{\prime}$ is the graph drawing obtained from $\mathfrak{q}$ by adding $n$ new edges, one in each face of $\mathfrak{q}$ as in the construction of $\phi\left(\mathfrak{q}, v_{0}\right)$. The edges of $\mathfrak{q}$ remain in $\mathfrak{q}^{\prime}$ and are called the old edges. Clearly, in view of the discussion of edge addition in Section $A, \mathfrak{q}^{\prime}$ is a map of genus $g$ : each face of $\mathfrak{q}$ is divided into two faces in $\mathfrak{q}^{\prime}$, either both of degree 3 , or one of degree 4 and one of degree 2 . Then $\phi\left(\mathfrak{q}, v_{0}\right)$ is obtained from $\mathfrak{q}^{\prime}$ by deleting the old edges and the vertex $v_{0}$. We have to check that deleting these old edges do not create a non simply connected face.

Since $\mathfrak{q}^{\prime}$ is a map, we can consider its dual $\mathfrak{q}^{\prime \prime}=\left(\mathfrak{q}^{\prime}\right)^{*}$. Let us orient the edges of $\mathfrak{q}^{\prime \prime}$ that are duals of the old edges of $\mathfrak{q}^{\prime}$ : an old edge of $\mathfrak{q}^{\prime \prime}$ whose dual edge has label $(i, i+1)$ is oriented so that it has the largest label $i+1$ on its right hand side (see Figure 5). The fake edges of the proof after Lemma 2 are exactly these oriented dual edges.

LEMMA 12. There is a unique outgoing edge leaving each vertex of $\mathfrak{q}^{\prime \prime}$, so that any cycle of oriented edges in $\mathfrak{q}^{\prime \prime}$ is in fact an oriented cycle. The only oriented cycle in $\mathfrak{q}^{\prime \prime}$ consists of the duals of the edges incident to $v_{0}$ in $\mathfrak{q}^{\prime}$.

PROOF. The first property follows immediately upon comparing our choice of orientation with the three types of faces of $\mathfrak{q}^{\prime}$, as represented on Figure 5 .

Now label each face of $\mathfrak{q}^{\prime}$ by the minimum of the labels of its incident vertices. The three cases represented in Figure 5 show that an oriented edge of $\mathfrak{q}^{\prime \prime}$ that crosses an edge of $\mathfrak{q}^{\prime}$ with label $(i, i+1)$ originates in a face with label $i$ and ends in a face with label $i$ or $i-1$ : hence an oriented cycle of edges of $\mathfrak{q}^{\prime \prime}$ must visit faces with non increasing labels, which are thus all equal. A closer look at the three cases furthermore shows that the only way to keep constant labels consists in turning counterclockwise around a fixed vertex of $\mathfrak{q}^{\prime}$. An oriented cycle visiting faces with label $i$ in $\mathfrak{q}^{\prime \prime}$ must therefore enclose exactly one vertex. This vertex has then label $i$ and all incident edges have label $(i, i+1)$. But, by definition of the distance labeling, there is only one vertex not incident to a vertex with a smaller label, the basepoint $v_{0}$.

Lemma 12 implies that the oriented edges of $\mathfrak{q}^{\prime \prime}$ form around $v_{0}$ an oriented cycle, to each vertex of which is attached a tree of edges oriented toward the cycle (see Figure 5).

Let us now consider the deletion of the old edges from $\mathfrak{q}^{\prime}$. Since the possible labels around a face of $\mathfrak{q}^{\prime}$ are $(i, i+1),(i, i+1, i+1)$ or $(i, i+1, i+2, i+1)$ for some $i \geq 0$, no face of $\mathfrak{q}^{\prime}$ can be incident twice to $v_{0}$. Hence, according to the second alinea of Lemma 10, removing the (old) edges incident to $v_{0}$ yields a simply connected face. 
Keeping track of this operation on the dual $\mathfrak{q}^{\prime \prime}$ amounts to the contraction of the oriented cycle into a single vertex: the remaining oriented edges of this modified dual then form a tree. According to Lemma 11, removing these remaining old edges from $\mathfrak{q}^{\prime}$ yields again a simply connected face. This proves that $\phi\left(\mathfrak{q}, v_{0}\right)$ is a well defined map on $S_{g}$, and that it has exactly one face: $\phi\left(\mathfrak{q}, v_{0}\right)$ is a $g$-tree with $n$ edges. Finally, the labels are positive integers and the new edges satisfy by construction the small variation condition so that $\phi\left(\mathfrak{q}, v_{0}\right)$ is a well labelled $g$-tree.

\section{B.2 The closure of a well labeled $g$-tree}

We describe here a mapping $\psi^{\prime}$, a priori different from $\psi$, but which we shall later prove to be the inverse of $\phi$. While the description of $\psi$ relied on Lemma 3, we explicitely describe how to add edges to a well labeled tree $\mathfrak{t}$ to produce its image $\psi^{\prime}(\mathfrak{t})$. In particular the forthcoming discussion implicitely contains a constructive proof of Lemma 3, and shows that $\psi^{\prime}=\psi$.

Given a well labeled map, let $c$ be a corner with label $\ell$ of a face $f$, and assume the minimal label around $f$ is $\ell_{0}<\ell$. The predecessor of $c$ is then defined as the first corner with label $\ell-1$ encountered after $c$ when going clockwise around the face $f$ : this definition makes sense as soon as the labels can decrease at most by one around any face, as is the case for well labeled maps. In particular in a well labeled $g$-tree, the predecessor is defined for any corner with label at least 2 , since there is a unique face with minimum label 1.

Let us now define the image $\psi^{\prime}(\mathfrak{t})$ of a $g$-tree $\mathfrak{t}$ with $n$ edges, and for this, assume that $\mathfrak{t}$ has $k$ corners with label 1 :

Phase 1. Add a new vertex $v_{0}$ in the unique face of $\mathfrak{t}$, and draw an edge between each corner of label 1 of $\mathfrak{t}$ and $v_{0}$, so that the unique face of $\mathfrak{t}$ is divided into $k$ simply connected faces, each incident once to $v_{0}$. The result is a map $\mathfrak{t}^{\prime}$ of genus $g$ with $k$ faces, denoted $f_{1}, \ldots, f_{k}$ in clockwise order around $v_{0}$. Let also $\vec{e}_{i}$ denote the arc with origin $v_{0}$ that has $f_{i}$ on its right-hand side.

By construction, each face $f_{i}$ of $\mathfrak{t}^{\prime}$ is incident once to $v_{0}$, it has one corner with label 0 . All corners of $\mathfrak{t}^{\prime}$ thus have a predecessor, except those incident to $v_{0}$.

Phase 2. In each face $f_{i}$ of $\mathfrak{t}^{\prime}$, insert iteratively a edge between each corner and its predecessor, in the counterclockwise order of corners around $f_{i}$, starting with the first corner after $v_{0}$. The result is a map $\mathfrak{t}^{\prime \prime}$ of genus $g$, containing the $n$ edges of $\mathfrak{t}$ and $2 n$ new edges.

As soon as an edge is added in $f_{i}$, this face is divided in the current map $\mathfrak{m}$ into smaller faces, and some corners are divided into smaller corners. However we will check that, due to the order of insertions, the corner $c$ of $f_{i}$ is still a corner of $\mathfrak{m}$ when it is considered, and that $\mathfrak{m}$ remains well labeled during all the insersion process. It thus makes sense to ask for the insertion of an edge between $c$ and its predecessor in $\mathfrak{m}$. Moreover the predecessor of $c$ in $\mathfrak{m}$ is a corner which can be naturally identified with the predecessor of $c$ in $\mathfrak{t}^{\prime}$, allowing for the shorthand statement at the beginning of Step 2.

Phase 3. Remove the edges of the map $\mathfrak{t}^{\prime}$ from the map $\mathfrak{t}^{\prime \prime}$ to get a pointed graph drawing $\psi^{\prime}(\mathfrak{t})=\left(\mathfrak{t}^{\prime \prime}-\mathfrak{t}^{\prime}, v_{0}\right)$. 
LEMMA 13. The pointed graph drawing $\psi^{\prime}(\mathfrak{t})$ is a pointed bipartite quadrangulation on $S_{g}$ with $2 n$ edges, and:

$$
\phi\left(\psi^{\prime}(\mathfrak{t})\right)=\mathfrak{t}
$$

ProOF. Let us first check that the construction is well defined. Let $f_{i}$ be one of the $k$ faces of $\mathfrak{t}^{\prime}$, let $c_{0}, c_{1}, c_{2}, \ldots, c_{q}$ be its corners in clockwise direction, starting with the corner of $v_{0}$, and let $\ell_{i}$ be the label of $c_{i}$. At the $p$ th step of the insertion in $f_{i}$, the edges associated with corners $c_{1}, \ldots, c_{p-1}$ have been inserted and the current map $\mathfrak{m}$ satisfy the following property, which is the invariant of our construction:

- Let $f$ be the face on the right hand side of the $\operatorname{arc} \vec{e}_{i}$ in $\mathfrak{m}$ : the corners of $f$ in counterclockwise order starting at $v_{0}$ are $c_{0}^{\prime}, c_{1}^{\prime}, c_{2}^{\prime}, \ldots, c_{\ell_{p-1}}^{\prime}, c_{p}, c_{p+1}, \ldots, c_{q}$, where each $c_{i}^{\prime}$ is a corner with label $i$ for $i=0, \ldots, \ell_{p-1}$.

In particular the corner $c_{p}$ of $f_{i}$ is a corner of $f$ in $\mathfrak{m}$ and its predecessor is the corner $c_{\ell_{p}-1}^{\prime}$ (beware that $c_{\ell_{p}-1}^{\prime}$ is different from $c_{\ell_{p-1}}^{\prime}$ unless $\ell_{p-1}=\ell_{p}-1$ ). The insertion of the edge $e$ between $c_{p}$ and its predecessor divides $f$ into 2 faces:

-On the right hand side of $\vec{e}$ (oriented from $c_{p}$ to $c_{\ell_{p}-1}^{\prime}$ ), remains a face incident to the edge $e_{i}$ with corners $c_{0}^{\prime}, \ldots, c_{\ell_{p}-2}^{\prime}, c^{\prime \prime}, c^{\prime \prime \prime}, c_{p+1} \ldots, c_{q}$ where $c^{\prime \prime}$ and $c^{\prime \prime \prime}$ have respectively label $\ell_{p}-1$ and $\ell_{p}$. This ensures the correctness of the invariant.

-On the left hand side of $\vec{e}$, remains a face of degree 2,3 , or 4 , depending on the value of $\ell_{p}-\ell_{p-1}$ (that is, on the variation on the edge between corners $p-1$ and $p$ along $f_{i}$ ). These 3 types of faces are illustrated by Figure 6 .

This concludes the proof that the construction is well defined.

In view of the 3 types of faces of $\mathfrak{t}^{\prime \prime}$, it is then clear that the edges of $\mathfrak{t}$ with label $(i, i)$ lie in faces of $\psi^{\prime}(\mathfrak{t})$ with labels $i, i-1, i, i-1$, and that the edges of $\mathfrak{t}$ with label $(i, i+1)$ lie in faces of $\psi^{\prime}(\mathfrak{t})$ with labels $i+1, i, i-1, i$ (see Figure 6 ). In particular each edge of $\mathfrak{t}$ lies in a face of $\psi^{\prime}(\mathfrak{t})$ of degree 4 . Hence $\psi^{\prime}(\mathfrak{t})$ is a quadrangulation, and it is bipartite because new edges always connect vertices of different parity.

Upon comparing the two cases in Figure 6 with the two cases for the creation of edges by $\phi$ in Figure 4, it clearly appears that in any face of $\psi^{\prime}(\mathfrak{t})$ the construction rules of $\phi\left(\psi^{\prime}(\mathfrak{t})\right)$ recreates the original edge of $\mathfrak{t}$, so that $\phi\left(\psi^{\prime}(\mathfrak{t})\right)=\mathfrak{t}$.

\section{B.3 Proof of the main theorem}

To complete the proof of Theorem 1 , it is sufficient to show that for every pointed bipartite quadrangulation $\left(\mathfrak{q}, v_{0}\right)$ and associated $g$-tree $\mathfrak{t}=\phi\left(\mathfrak{q}, v_{0}\right)$, one has $\psi^{\prime}(\mathfrak{t})=$ $\left(\mathfrak{q}, v_{0}\right)$. The key step is the following lemma:

LEMMA 14. Let $\left(\mathfrak{q}, v_{0}\right)$ be a pointed bipartite quadrangulation, let $\mathfrak{t}=\phi\left(\mathfrak{q}, v_{0}\right)$ be the associated $g$-tree. Let $\mathfrak{q}^{\prime}=\mathfrak{q} \cup \mathfrak{t}$, that is $\mathfrak{q}^{\prime}$ is the map in which the edges of $\mathfrak{t}$ have been added to $\mathfrak{q}$, and let $\mathfrak{t}^{\prime \prime}=\mathfrak{t} \cup \psi^{\prime}(\mathfrak{t})$, that is the map in which the edges of $\psi^{\prime}(\mathfrak{t})$ have been added to $\mathfrak{t}$. Both $\mathfrak{q}^{\prime}$ and $\mathfrak{t}^{\prime \prime}$ contain the $g$-tree $\mathfrak{t}$.

If there is an edge $e$ of $\mathfrak{q}$ that connects two corners of $\mathfrak{t}$ in $\mathfrak{q}^{\prime}$ (resp. a corner of $\mathfrak{t}$ and $\left.v_{0}\right)$, then there is in $\mathfrak{t}^{\prime \prime}$ an edge between the same corners of $\mathfrak{t}$ (resp. between the same corner of $\mathfrak{t}$ and $\left.v_{0}\right)$.

Proof. If $e$ is adjacent to $v_{0}$, then it is immediately replaced in $\psi^{\prime}(\mathfrak{t})$ during the first phase of the construction, and the conclusion of the lemma holds. 


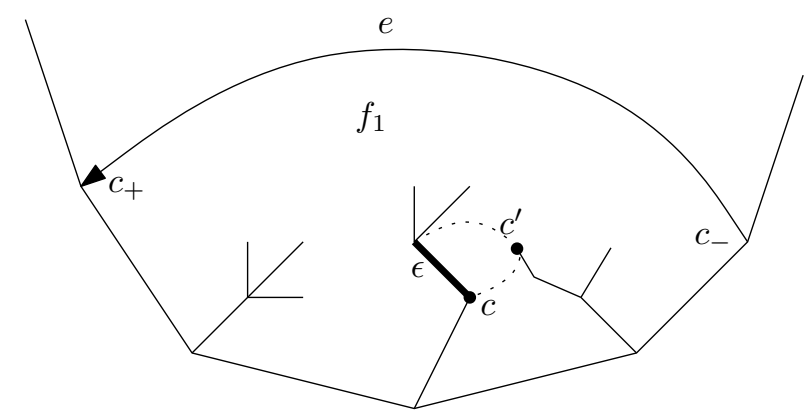

Fig. 9. The proof of Lemma 14

Let us thus consider an edge $e$ of $\mathfrak{q}$ not adjacent to $v_{0}$, and let us show that it is recovered in $\mathfrak{t}^{\prime \prime}$. Let $\mathfrak{t}^{\prime}$ be the map obtained by adding to $\mathfrak{t}$ the vertex $v_{0}$ and all the edges adjacent to $v_{0}$ in $\mathfrak{q}: \mathfrak{t}^{\prime}$ is a submap of $\mathfrak{q}^{\prime}$, and each face of $\mathfrak{t}^{\prime}$ is adjacent to exactly one corner with label 0 . Since $\mathfrak{t}^{\prime}$ spans all the vertices of $\mathfrak{q}^{\prime}$, e must lie in some face $f$ of $\mathfrak{t}^{\prime}$, cutting it into two new faces. Call $f_{1}$ the one of these two faces which does not contains the corner labeled 0 , and orient $e=\left(c_{-}, c_{+}\right)$in such a way that it has $f_{1}$ on its left. Here, $c_{-}$and $c_{+}$are two corners of $\mathfrak{t}$, but up to a slight abuse of notations, can be considered as corners of $f_{1}$ as well.

We claim that $c_{+}$is the unique corner of minimal label in $f_{1}$. Indeed, assume that the minimal label in $f_{1}$ is reached at some corner $c \neq c_{+}$, and let $\ell_{c}$ be its label. Let $\epsilon$ be the edge of $\mathfrak{t}$ leaving $c$ in clockwise direction around $f_{1}$. By the rules of construction of $\mathfrak{t}, \epsilon$ has been obtained from a face of $\mathfrak{q}$ that contains a vertex $c^{\prime}$ of label $\ell_{c}-1$ on the right of $\epsilon$. Since $\mathfrak{q}^{\prime}$ is a well defined map, with no vertex strictly inside $f_{1}$, the vertex $c^{\prime}$ must lie on the border of $f_{1}$ (see Figure 9 ). This contradicts the minimality of the label $\ell_{c}$ in $f_{1}$.

Hence $c_{+}$is the unique corner of minimal label in $f_{1}$. Since $e$ is an edge of $\mathfrak{q}$ this implies that $\ell_{c_{-}}=\ell_{c_{+}}+1$, and that $c_{+}$is the predecessor of $c_{+}$in $\mathfrak{t}: c_{+}$and $c_{-}$are linked by an edge in $\mathfrak{t}^{\prime \prime}$.

From the lemma, the set of edges of $\mathfrak{q}$ is naturally included in the set of edges of $\psi^{\prime}(\mathfrak{t})$. Now, by construction, the number of edges in $\psi^{\prime}(\mathfrak{t})$ is equal to the number of edges in $\mathfrak{q}$. Hence $\psi^{\prime}(\mathfrak{t})$ and $\mathfrak{q}$ have exactly the same set of edges, and thus are equal. This completes the proof of Theorem 1. 